\title{
Contrasting subduction-exhumation paths in the blueschists of the Anarak Metamorphic Complex (Central Iran)
}

\author{
S. ZANCHETTA*†, N. MALASPINA*,A.ZANCHI*,L.BENCIOLINIt, \\ S. MARTIN, H.R. JAVADIף \& M. KOUHPEYMA \\ *Dipartimento di Scienze dell'Ambiente e della Terra, Piazza della Scienza 4, 20126 Milano, Italy \\ tDipartimento di Fisica, Chimica e Ambiente, Università degli Studi di Udine, Italy \\ $\S$ Dipartimento di Geoscienze, Università degli Studi di Padova, Italy \\ qGeological Survey of Iran, Azadi Square, Meraj Avenue, 13185-1494 Tehran, Iran
}

(Received 2 November 2016; accepted 17 February 2017; first published online 3 April 2017)

\begin{abstract}
The Anarak Metamorphic Complex, localized in Central Iran, is a fossil accretionary wedge composed of several tectonometamorphic units. Some of these, the Chah Gorbeh, the Morghab and the Ophiolitic complexes, contain mafic rocks that have been metamorphosed at high-pressurelow-temperature conditions. Such units have been stacked together and later refolded during the final stages of exhumation. Structural analysis at the mesoscale recognized at least three deformation events. Microstructural analyses, mineral chemistry and thermodynamic modelling reveal that the mafic schists followed contrasting $P-T$ paths during their tectonometamorphic evolutions. In the schists of the Chah Gorbeh and Ophiolitic complexes an early greenschist-facies stage was later overprinted by blueschist-facies phase assemblages with suggested peak conditions of $390-440{ }^{\circ} \mathrm{C}$ at $0.6-0.9 \mathrm{GPa}$ for the meta-basalt within the Ophiolitic Complex and $320-380^{\circ} \mathrm{C}$ at $0.6-0.9 \mathrm{GPa}$ for the blueschists of the Chah Gorbeh Complex. $P-T$ conditions at metamorphic peak were 410 $450^{\circ} \mathrm{C}$ at $0.78-0.9 \mathrm{GPa}$ for the Morghab blueschists, but they are reached before a greenschist-facies re-equilibration. Compositional zoning of amphiboles and epidotes of this greenschist-facies stage suggests a renewed pressure increase at the end of this metamorphic stage. Based on these data we reconstructed a clockwise $P-T$ path for the Morghab mafic schists and a counter-clockwise path for the Chah Gorbeh blueschists and ophiolitic meta-basalts. Such contrasting metamorphic evolutions of tectonic units that were later accreted to the same wedge are indicative of the complex tectonic dynamics that occur within accretionary-subduction complexes.
\end{abstract}

Keywords: Central Iran, mafic blueschists, accretionary wedge, $P-T$ evolution

\section{Introduction}

The occurrence of mafic blueschists is considered one of the main points of evidence for oceanic subduction accompanied by the formation of an accretionary prism between the upper and the subducting plate (e.g. Ernst, 1973). Blueschist-facies rocks are mainly found to occur as large coherent terranes or as isolated blocks within a serpentinitic or pelitic matrix in tectonic mélange complexes (Hsu, 1968).

The $P-T$ conditions at metamorphic peak and the subduction-exhumation paths followed by blueschistfacies rocks strongly depend on the thermal regime during subduction, which in turn depends on the age and structure of the subducting plate (e.g. Cloos \& Shreve, 1988a,b), and on the time at which the rocks are subducted with respect to subduction initiation (i.e. initial versus late-subducted blueschists). Depressed thermal regimes usually develop only after a few million years following subduction initiation (Gerya \& Stockhert, 2006), and rock units that are subducted later may escape a first heating stage that is commonly experienced by units that are underplated in the nas-

$\dagger$ †uthor for correspondence: stefano.zanchetta@unimib.it cent accretionary wedge at subduction initiation (Ukar, 2012).

The common occurrence within a single accretionary complex of blueschists that have reached different depths and followed different subduction-exhumation paths (e.g. Agard et al. 2010) testifies to the complexity of the tectonic and thermal structure of an evolving accretionary wedge.

In Central Iran, the Anarak Metamorphic Complex (AMC) is interpreted as a fossil accretionary prism (Sharkovski, Susov \& Krivyakin, 1984; Bagheri \& Stampfli, 2008) developed in the framework of the northward subduction of the Palaeo-Tethys beneath the southern Eurasian margin (e.g. Şengör, 1979). The AMC consists of several units, some of which were metamorphosed at blueschist-facies conditions (Zanchi et al. 2009, 2015). The difference in lithological composition, metamorphic peak conditions and age of metamorphism led some authors to consider the AMC as a composite complex, formed during tectonic events that were separated in time and space.

In this paper, we address the petrographic and textural features of mafic blueschists of the AMC, in order to reveal the recrystallization stages and the deformation-metamorphism relationships in the Chah 


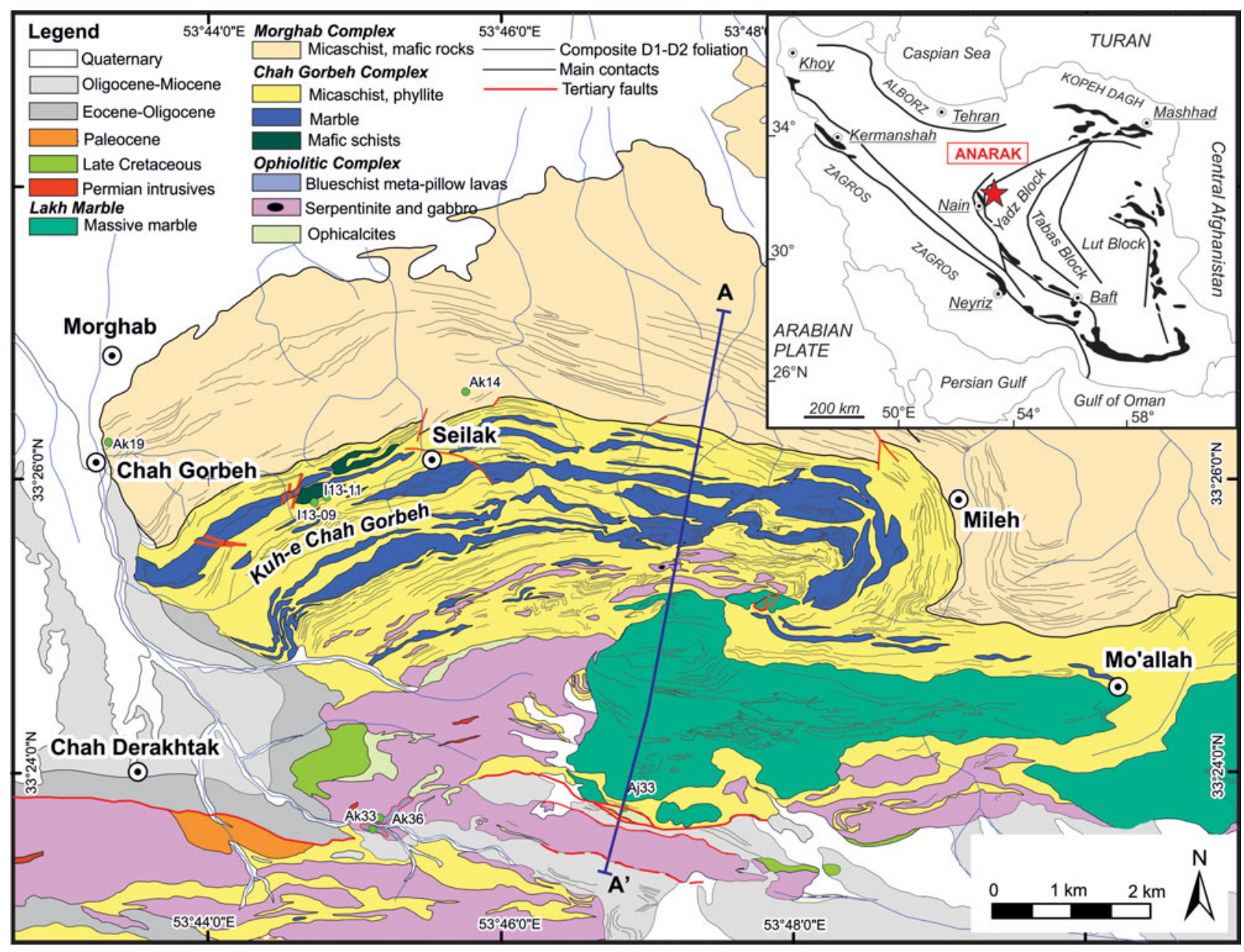

Figure 1. (Colour online) Geological map of the Anarak Metamorphic Complex with location of analysed samples. The trace of the geological cross-section in Figure 2 is here indicated. Modified after Zanchi et al. (2015).

Gorbeh, Morghab and Ophiolitic complexes. We also performed thermodynamic modelling in order to estimate the $P$ and $T$ conditions of each metamorphic stage recognized in the analysed samples.

The whole dataset of petrographic, microstructural and thermodynamic data is then used to define the $P-T$ trajectories of mafic blueschists from the three units. The results are then discussed in the context of accretionary wedge dynamics and AMC tectonic significance.

\section{Geological setting}

The AMC crops out in Central Iran, to the east of Nain (Fig. 1). It consists of several subunits exposed for c. $150 \mathrm{~km}$ in an E-W-trending belt, stacked together during polyphase metamorphic and deformation events (Sharkovski, Susov \& Krivyakin, 1984; Bagheri \& Stampfli, 2008; Zanchi et al. 2009; Buchs et al. 2013; Zanchi et al. 2015). The AMC is in tectonic contact with other metamorphic complexes and sedimentary successions of various ages and palaeogeographic affinities (Fig. 1). To the west, the Great Kavir - Doruneh fault system represents the contact between the AMC and the Cretaceous ophiolites that border the entire Central-East Iranian Microcontinent (Ghasemi \& Talbot, 2006). The southern limit of the AMC coincides with the NW border of the Yadz block whereas to the east the Jandaq complex, a magmatic-metamorphic unit with a poorly defined age (likely Carboniferous or pre-Carboniferous, Bagheri \& Stampfli, 2008; Berra et al. 2017), is exposed. Finally, to the north of the AMC, the non-metamorphic Nakhlak ophiolite-sedimentary complex occurs (Balini et al. 2009). The contact between the AMC and the Nakhlak complex is not exposed, so the relationships existing between the complexes are not clear (Balini et al. 2009; Zanchi et al. 2009).

The AMC is composed of several subunits which display heterogeneous structural and metamorphic evolutions (Sharkovski, Susov \& Krivyakin, 1984; Bagheri \& Stampfli, 2008; Zanchi et al. 2009, 2015). The Morghab Complex largely consists of metapelitic schists with abundant interleaved quartzite and metabasite layers (Zanchi et al. 2015). Minor meta-volcanic rocks and amphibole-bearing gneisses occur in the Kuh-e Pol-e Khavand area (Fig. 1). 


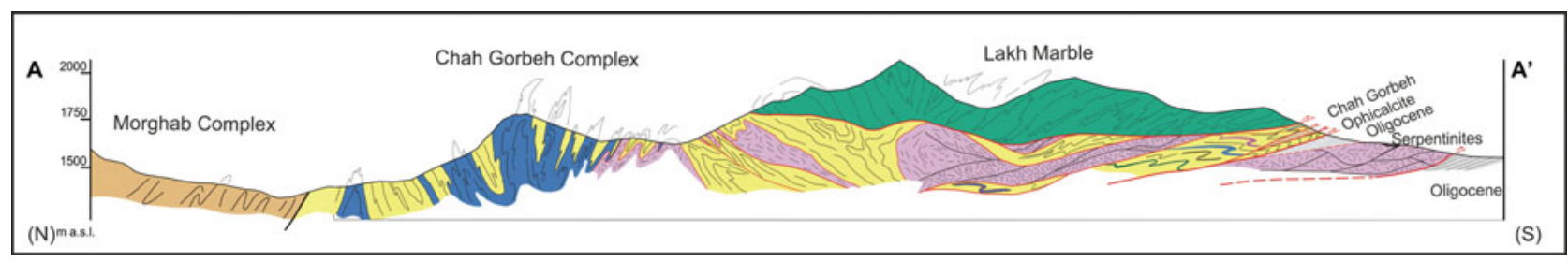

Figure 2. (Colour online) Schematic cross-section showing the main structural features of the AMC and the tectonic relationships between the CGC, MC and OC complexes and the overlying Lakh Marble. Modified after Zanchi et al. (2015).

We will focus in this paper on the three main units that compose the AMC: the Chah Gorbeh (CGC), Morghab (MC) and Ophiolitic (OC) complexes (Zanchi et al. 2015).

Quartzite-rich phyllites, micaschists and metabasites interlayered with thick metacarbonate layers (Figs 1,2) form the CGC. Serpentinite lenses and layers occur in the southern part of the CGC.

The MC occurs to the north and east of the CGC. The MC has a more monotonous lithological composition, chiefly consisting of metapelites (phyllites and micaschists) with minor intercalations of quartzites, metabasites and thin marble layers. Regional folds with steep fold axes affected both the $\mathrm{MC}$ and CGC; this feature is particularly evident at the eastern end of the CGC (Fig. 1). To the south and southeast of the CGC an 'ophiolitic' complex (OC) occurs (Bagheri \& Stampfli, 2008; Zanchi et al. 2015). This complex largely consists of serpentinites and partly serpentinized harzburgites, where relics of the primary mineral assemblages and textures are scarcely preserved. In the central area of the OC some metabasite lenses have been observed (Fig. 1). Such lenses consist of metamorphosed basalts with well-preserved pillow structures. The OC is intensively folded with minor shear zones localized both along lithological contacts as well as within serpentinites.

The CGC, MC and OC units of the AMC are overthrust by a large thrust sheet of metacarbonates, the Lakh Marble (Bagheri \& Stampfli, 2008; Zanchi et al. 2015). These strongly recrystallized carbonates display an uneven distribution of deformation, with isoclinally folded areas coexisting with more preserved parts with sedimentary structures such as bedding, intraformational paraconglomerates (i.e. rich in matrix) and fossils. The age of the Lakh Marble is substantially unknown, although a late Palaeozoic age has been proposed based on indirect evidence (Bagheri \& Stampfli, 2008). Recent re-examination of the presumed Lower Cambrian Archeocyatid faunas described by Russian authors in the past (Mel'nikov et al. 1986) resulted in the identification of the demosponge genus Rankenella (Kruse \& Zhuralev, 2008) in the Kabudan Marble, which is a correlative of the Lakh Formation. These latter authors thus suggested a possible correlation with the Upper Cambrian Mila Formation of the Alborz Mountains, which implies a Gondwanan affinity for this unit.
Time constraints on the age of the high-pressurelow-temperature (HP-LT) metamorphism of the AMC are still lacking. A minimum age is provided by undeformed and non-metamorphosed trondhjemitic dykes and stocks that intruded the AMC (Torabi, 2012). Late Permian (Bagheri \& Stampfli, 2008) to Sakmarian ages (Zanchi et al. 2015) have been provided for these intrusive rocks, suggesting that deformation and metamorphism of the AMC is Early Permian or older.

The AMC is currently interpreted as an allochthonous crustal fragment that was part of an accretionary wedge developed along the southern Eurasian margin, in the hangingwall of the Palaeo-Tethys subduction zone (Zanchi et al. 2015). In this interpretation, the formation of the AMC is considered to have preceded the collision of the Iran plate with Eurasia that led to the Cimmerian orogenic event.

\section{Meso-structural aspects of the AMC}

We summarize here the main structural features of the metabasite-bearing units of the AMC as observed in the field. For a more exhaustive description of the structural setting and evolution of the AMC see Zanchi et al. (2015).

\section{3.a. Morghab Complex}

The MC displays a complex structural pattern formed in response to superposed deformation events, the first two out of three associated with syn-metamorphic isoclinal folding. $\mathrm{D}_{1}$ structures are now preserved at the mesoscale only as relicts within the $\mathrm{S}_{2}$ regional foliation. Rootless fold hinges are somewhat visible in quartzites and quartz-rich schists, whereas $\mathrm{D}_{1}$-related structures are visible only at the microscale in metabasites. Mineral phase assemblages in textural equilibrium with $\mathrm{D}_{1}$ structures point to HP-LT conditions during $\mathrm{D}_{1}$ (Zanchi et al. 2015).

The $\mathrm{D}_{2}$ deformation event is characterized by isoclinal folding developed both at the meso- and regional scale. The axial plane foliation, $\mathrm{S}_{2}$, of this fold system is the most pervasive fabric element within all the rock types that form the MC. Besides the $\mathrm{S}_{2}$ foliation a well-developed stretching lineation defined by elongated quartz and chlorite has been frequently observed. The $S_{2}$ foliation is 

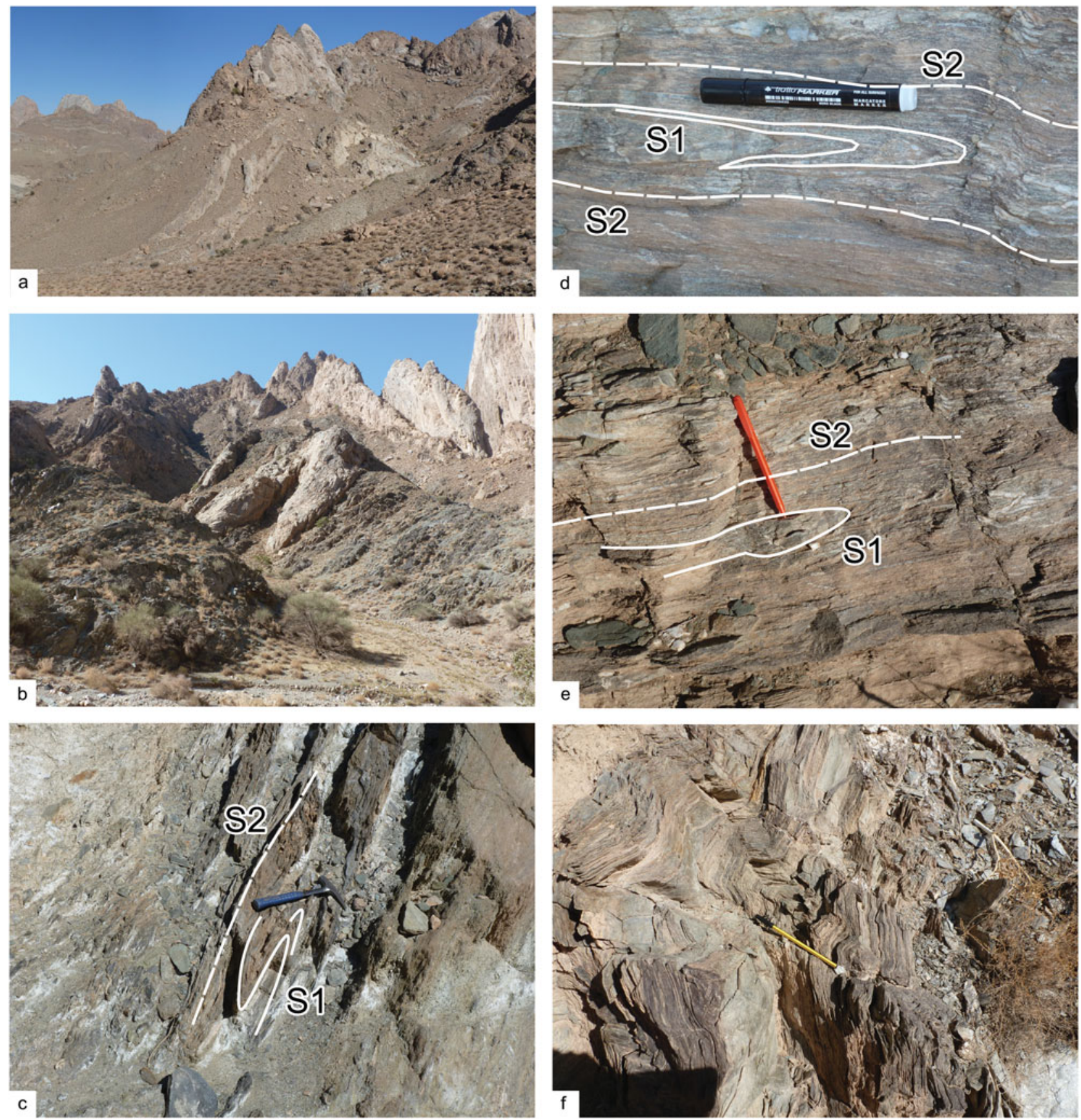

Figure 3. (Colour online) (a) Marble layers interleaved with metapelites and mafic schists of the CGC. (b) A fold hinge with a nearly vertical fold axis in the metacarbonates of the CGC. (c) $S_{1}$ relict foliation preserved within the regional $S_{2}$ foliation in paragneiss. Hammer for scale is $33 \mathrm{~cm}$ long. (d) $S_{1}$ relics within schists of the MC. Pen for scale is $14 \mathrm{~cm}$ long. (e) Superposed $D_{1}-D_{2}$ folds in the $\mathrm{MC}$ rocks. Pen for scale is $15 \mathrm{~cm}$ long. (f) Open to medium-closed $\mathrm{D}_{3}$ folds with an axial fracture cleavage. Pen for scale is $15 \mathrm{~cm}$ long.

concordant with lithological contacts within the MC and between the $\mathrm{MC}$ and adjoining units. Fold axes related to $D_{2}$ folds range from sub-horizontal to nearly vertical, suggesting that a successive deformation event affected the complex. This event, $\mathrm{D}_{3}$, is responsible for the development of medium-tight to closed folds observable both at the meso- and regional scale. $D_{3}$ folds deflecting the $S_{2}$ foliation and affecting the contact between the CGC and MC complexes are clearly reported in the geological-structural map of Figure 1 and characterize the present-day structural setting.

\section{3.b. Chah Gorbeh Complex}

As in the MC the oldest recognized structures are poorly visible at the mesoscale. They are recognizable as relict $S_{1}$ foliation intrafolial in the dominant $S_{2}$ one (Fig. 3c, d). Rootless fold hinges seldom occur in metacarbonates and metapelites/metapsammites. The $\mathrm{S}_{1}$ foliation is poorly visible at the mesoscale but clearly evident at the microscale where it is defined by a greenschist-facies mineral assemblage (Fig. 5).

The $\mathrm{D}_{2}$ deformation event formed isoclinal folding associated with a pervasive axial plane foliation, 

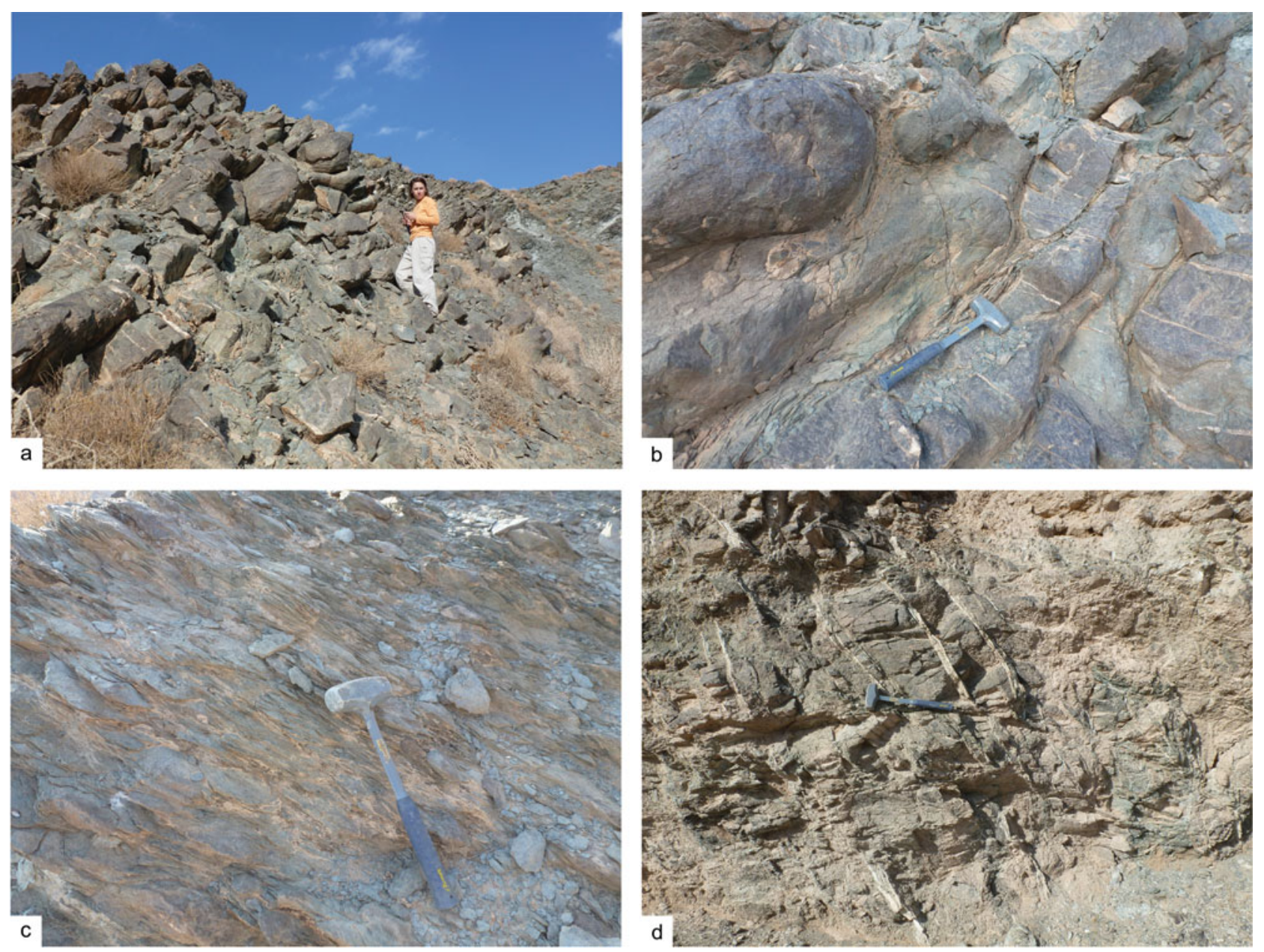

Figure 4. (Colour online) (a) Field aspect of pillow structure within meta-basalts of the OC; person for scale is $c$. $1.55-1.6 \mathrm{~m}$ tall. (b) Sheared domains developed at the rims of pillow. (c) Mylonitic foliation defined by blueschist-facies minerals at the contact between meta-basalts and serpentinites. (d) Calcite and quartz veins cross-cutting sheared serpentinites. Hammer for scale is $40 \mathrm{~cm}$ long.

$\mathrm{S}_{2}$, defined by Na-amphibole (Zanchi et al. 2015) that points to HP-LT metamorphism during the $\mathrm{D}_{2}$ event. The different $P-T$ conditions at which the regional foliation formed in the MC (greenschist facies) and CGC (blueschist facies) suggests that, even if the main foliations of both units are almost parallel to their contacts, they could not be related to a single deformation event that affected the $\mathrm{MC}$ and $\mathrm{CGC}$ together.

The contact between the CGC and serpentinites of the $\mathrm{OC}$ is marked by ductile shear zones (Fig. 2), sometimes with lenses of ophicalcites and impure marbles, where Na-amphibole is stable along the mylonitic foliation. This suggests that the $\mathrm{MC}$ and serpentinites were tectonically coupled at HP-LT conditions. In a few localities, in the southern part of the CGC, serpentinites are deformed by $\mathrm{D}_{2}$ isoclinal folds together with rocks of the CGC. This observation suggests that the OC and serpentinites share a common tectonometamorphic evolution from the $\mathrm{D}_{2}$ event onwards.

\section{3.c. Ophiolitic Complex}

We refer here to the whole complex of ultramafic rocks and blueschist-facies meta-basalts, the same that were distinguished by Zanchi et al. (2015) into two units: 'ultramafic rocks' and 'blueschist pillow meta-basalts'. The contacts between the ultramafic rocks and metabasalts are always tectonic (Fig. 4c), so a direct correlation among structures observed in the two units is not feasible, justifying the distinction proposed by Zanchi et al. (2015).

Ultramafic rocks occur in two distinct structural settings: (1) as tectonic slices within the southern part of the CGC, as previously described; (2) as a continuous unit, forming complex imbricated thrust sheets, in a lower structural position with respect to the CGC (Fig. 2). Peridotites of type (1) are almost completely serpentinized and intensively sheared with foliations subparallel to the $\mathrm{S}_{2}$ of the CGC and to tectonic contacts. Peridotites of type (2) show a patchy distribution of deformation with isolated lenses conserving a massive texture with preserved olivine, orthopyroxene and spinel crystals. This mineral assemblage indicates a harzburgitic composition for the ultramafic rocks of the AMC. Such massive domains are wrapped by sheared domains where a pervasive schistosity is associated with an almost complete serpentinization of harzburgites. 

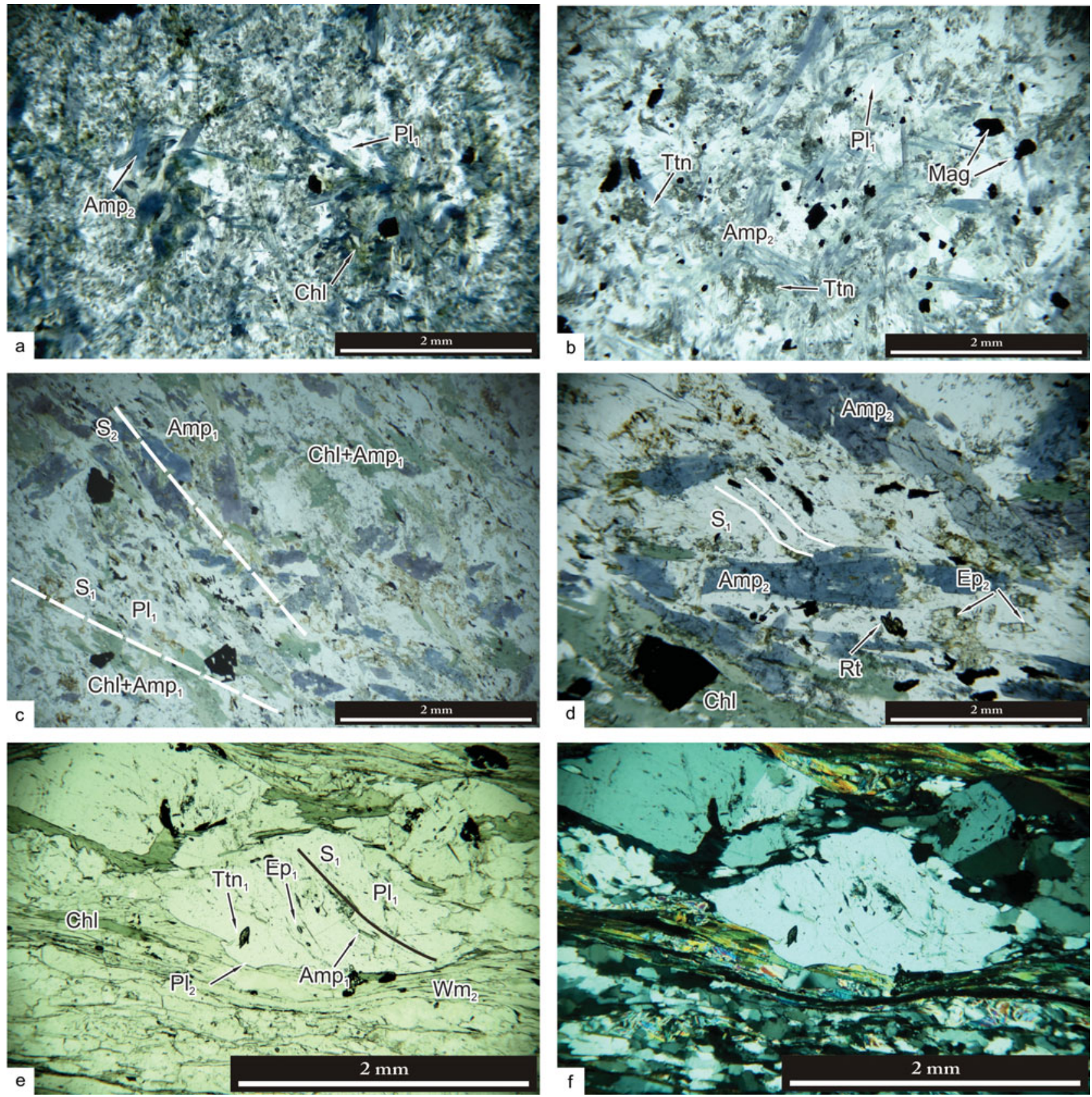

Figure 5. (Colour online) Microstructural features of mafic schists. (a, b) Static growth of blue amphibole $\left(\right.$ Amp $\left._{2}\right)$, magnetite and titanite $\left(\mathrm{Ttn}_{2}\right)$ on existing greenschist-facies assemblage in meta-basalts. (c, d) Syn-kinematic Na-amphibole $\left(\mathrm{Amp}_{2}\right)$ and epidote $\left(\mathrm{Ep}_{2}\right)$ aligned parallel to $S_{2}$ within mafic schists of the CGC. Relics of a greenschist-facies $S_{1}$ foliation are best preserved within plagioclase porphyroblasts. $(\mathrm{e}, \mathrm{f})$ Blueschist-facies phase assemblage aligned defining the internal foliation of plagioclase porphyroblasts $\left(\mathrm{Pl}_{2}\right)$ in retrogressed blueschists of the MC.

Meta-basalts with well-preserved pillow structures (Fig. 4a, b) occur ESE of Chah Derakhtak (Fig. 1). Such slices form part of a complex Sverging thrust stack also involving serpentinites and ophicalcites (Sharkovski, Susov \& Krivyakin, 1984; Zanchi et al. 2009, 2015). Except within shear zones, where pillows are partially to almost completely transposed (Fig. 4c), the meta-basalts are substantially undeformed. A poorly defined foliation is distinguishable only at the microscale, defined by greenschist-facies mineral assemblage (see next section).

\section{Microstructural and metamorphic evolution}

Microstructural analyses on selected samples of mafic rocks from the three tectonic units were performed to decipher the deformation-metamorphism relationships in each unit. The multi-stage structural evolution reconstructed at the mesoscale has also been recognized at the microscale. A brief overview of the chemical composition of key minerals occurring at distinct microstructural positions is provided here, whereas the reader is referred to the next section for a complete description of the AMC rocks' mineral 
chemistry. Mineral abbreviations used in the text are reported in Table 1.

\section{4.a. Morghab Complex}

The mineralogy of mafic schists of the MC is made of (Table 1): amphibole, plagioclase, epidote, chlorite, white mica, titanite, quartz, magnetite and apatite. Secondary calcite occurring in veins and microcracks post-dating the main foliation has been observed in some samples.

Metabasites of the MC are fine grained with the main fabric element at the microscale represented by a well-developed foliation. Cores of epidote $\left(\mathrm{Ep}_{2}\right)$ and albitic plagioclase $\left(\mathrm{Pl}_{1}\right)$ porphyroblasts partially predate the main foliation. Aligned inclusion trails have been observed both within $\mathrm{Pl}_{2}$ and $\mathrm{Ep}_{2}$. They consist of blue Na-rich amphibole $\left(\mathrm{Amp}_{1}\right)$, white mica $\left(\mathrm{Wm}_{1}\right)$, titanite $\left(\mathrm{Ttn}_{1}\right)$, quartz, albite $\left(\mathrm{Pl}_{1}\right)$ and epidote $\left(\mathrm{Ep}_{1}\right) . \mathrm{Pl}_{1}$ and $\mathrm{Ep}_{1}$ inclusions occur within $\mathrm{Ep}_{2}$ and $\mathrm{Pl}_{2}$, respectively. The main foliation, which corresponds to the regional $S_{2}$ foliation as recognized by field structural analysis, wraps around plagioclase and epidote porphyroblast cores. The outer rims of both $\mathrm{Ep}_{2}$ and $\mathrm{Pl}_{2}$ seem to overgrow the foliation, so we consider the porphyroblast rims to be syn- to post-kinematic with respect to $S_{2}$. The $S_{2}$ foliation is defined by the shape preferred orientation (SPO) of $\mathrm{Amp}_{2}+\mathrm{Chl}+\mathrm{Wm}_{2}+\mathrm{Ttn}_{2}$. Amp $\mathrm{Accurs}_{2}$ in the form of tiny to fine-grained crystals with a subidiomorphic to idiomorphic (prismatic) habit. They frequently contain inclusions of quartz, chlorite and plagioclase. $\mathrm{Ttn}_{2}$ forms trails of (sub)millimetric (max. $1.5 \mathrm{~mm}$ ) crystals aligned parallel to $S_{2}$. Ttn 2 crystals always have the typical lozenge-shaped prismatic habit of titanite with the major axis elongated parallel to the $\mathrm{S}_{2}$ foliation. Both $\mathrm{Ttn}_{2}$ and $\mathrm{Amp}_{2}$ have been observed as inclusions in $\mathrm{Ep}_{2}$ porphyroblasts rims, suggesting that epidote crystal growth outlasted the $\mathrm{D}_{2}$ deformation event. A colour zoning is frequently observed in $\mathrm{Amp}_{2}$ : minerochemical analyses (see next section) highlighted a $\mathrm{Na}$-increase from the core to the rim of $\mathrm{Amp}_{2}$ crystals. A similar compositional zoning also occurs in $\mathrm{Ep}_{2}$ porphyroblasts, with rims enriched in $\mathrm{Fe}^{3+}$ with respect to the cores. This suggests a pressure increase during the late stages of the $\mathrm{D}_{2}$ deformation event. Amp $\mathrm{p}_{2}$ crystals also occur as inclusions within $\mathrm{Ep}_{2}$ rims.

Summarizing the metamorphic evolution individuated for the mafic schists of the MC we can define three successive parageneses: (i)Blueschist facies $\left(\right.$ pre- $\left.\mathrm{S}_{2}\right): \mathrm{Amp}_{1}+\mathrm{Wm}_{1}+\mathrm{Ttn}_{1}+\mathrm{Ep}_{1}+\mathrm{Pl}_{1}+\mathrm{Qtz}$; (ii) Greenschist facies $\left(\operatorname{syn}-\mathrm{S}_{2}\right): \mathrm{Amp}_{2}($ core $)+\mathrm{Chl}+$ $\mathrm{Wm}_{2}+\mathrm{Ep}_{2}$ (core) $+\mathrm{Pl}_{2}+\mathrm{Ttn}_{2}+\mathrm{Qtz}$; (iii) Greenschist with $P$ increase (late-, post- $\left.\mathrm{S}_{2}\right): \mathrm{Amp}_{2}(\mathrm{rim})+$ $\mathrm{Ep}_{2}($ rim) .

\section{4.b. Chah Gorbeh Complex}

The mineralogy of metabasites of the CGC consists of: amphibole, plagioclase, chlorite, white mica, epidote,

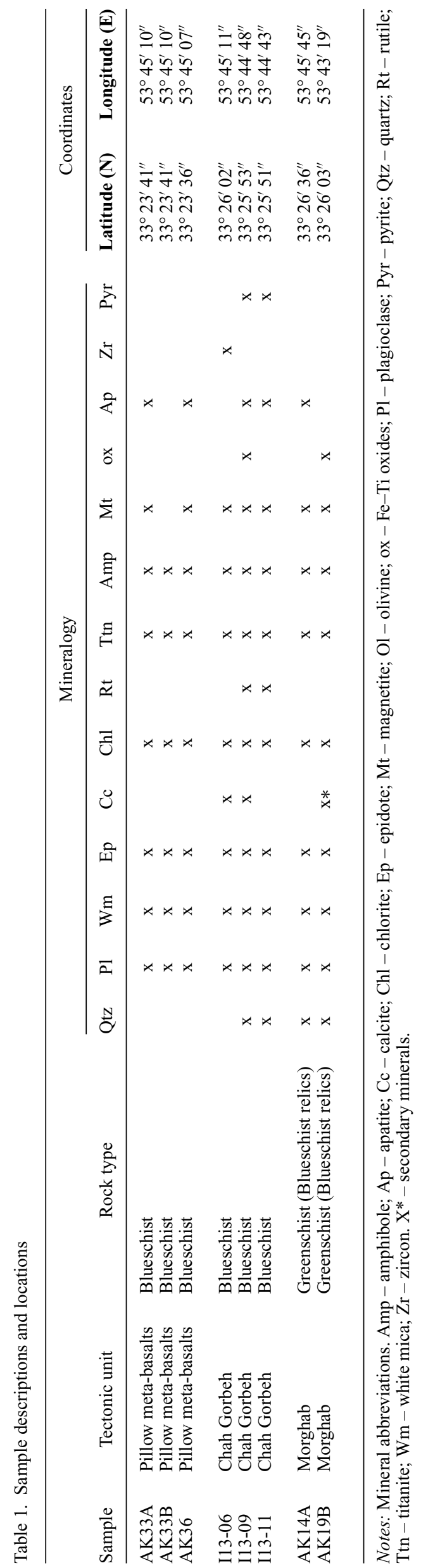


Morghab Complex
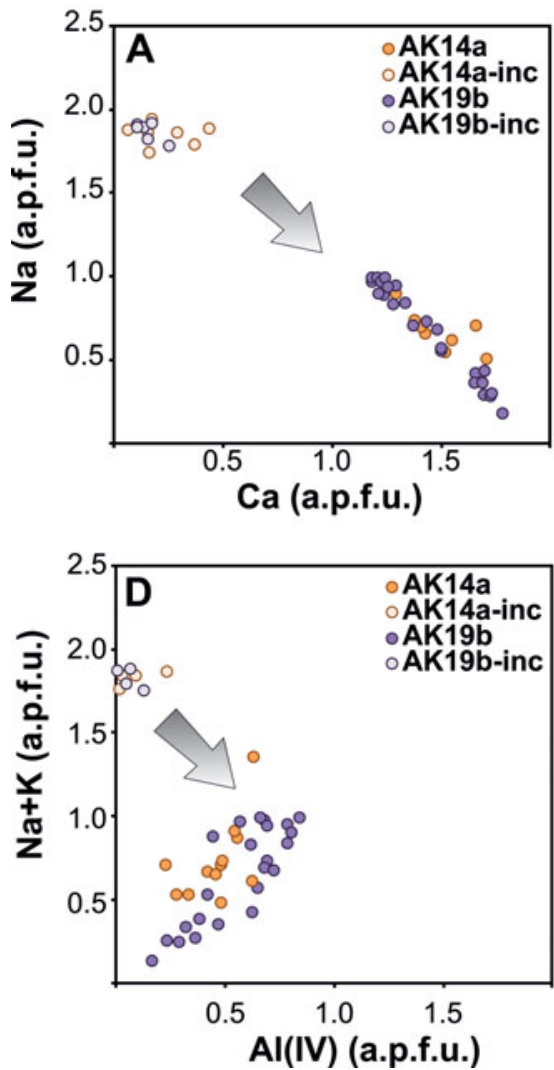

Chah Gorbeh Complex
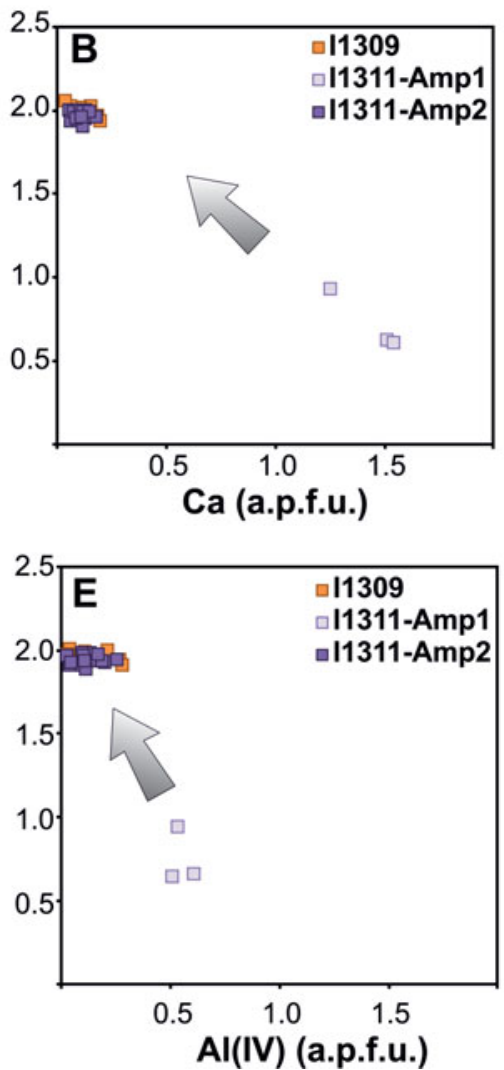

Ophiolites
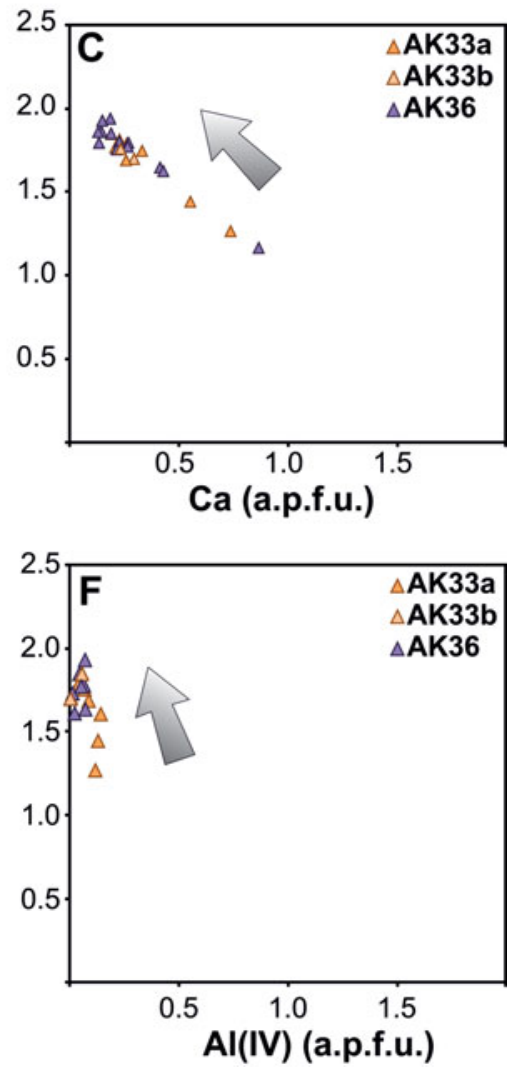

Figure 6. (Colour online) Representative amphibole compositions of mafic schists for the CGC, MC and OC. Arrows indicate the evolution direction (old to young) as recognized by microstructural analyses.

quartz, titanite, rutile, calcite, apatite, zircon and pyrite (Table 1).

The textural features of the mafic schists from the CGC closely resemble the ones described for the rocks of the MC. The development of a primary foliation is preceded by plagioclase $\left(\mathrm{Pl}_{1}\right)$ and epidote $\left(\mathrm{Ep}_{1}\right)$ porphyroblast growth, with a peculiar mineral phase assemblage of oriented inclusions. However, the metamorphic evolution is discordant, as we will describe below.

An internal foliation $\left(\mathrm{S}_{1}\right)$ made by the preferred orientation of $\mathrm{Ttn}_{1}+\mathrm{Pl}_{1}+\mathrm{Qtz}+\mathrm{Amp}_{1}$ inclusions is highlighted within $\mathrm{Pl}_{1}$ and $\mathrm{Ep}_{1}$ porphyroblasts. Amp is actinolitic hornblende (Fig. 6; Table 3), suggesting equilibration at relatively low $P$ under greenschistfacies conditions. The main foliation $\left(S_{2}\right)$ is defined by the SPO of $\mathrm{Chl}+\mathrm{Ab}+\mathrm{Wm}_{1}+\mathrm{Ep}_{2}+\mathrm{Rt}+\mathrm{Amp}_{2}$. Blue-coloured $\mathrm{Amp}_{2}$ is ferroglaucophane (Table 3) in composition and displays clear cores without inclusions, coupled with inclusion-rich rims, where $\mathrm{Wm}_{1}$, $\mathrm{Chl}$ and $\mathrm{Ep}_{2}$ aligned parallel to $\mathrm{S}_{2}$ occur. $\mathrm{Ep}_{2}$ is $\mathrm{Fe}-$ enriched (Fig. 6) with respect to $\mathrm{Ep}_{1}$, pointing to a $P$ increase from the $D_{1}$ to $D_{2}$ deformation/metamorphic event. Tiny magnetite crystals occur as trails aligned parallel to $\mathrm{S}_{2}$. Rutile, usually overgrown by rims of titanite $\left(\mathrm{Ttn}_{2}\right)$, has also been observed only outside $\mathrm{Pl}_{1}$ and $\mathrm{Ep}_{1}$ porphyroblasts. Therefore, its growth and incomplete substitution by $\mathrm{Ttn}_{2}$ could be assigned to the syn- to post- $\mathrm{S}_{2}$ metamorphic stage.
Two main metamorphic stages can be defined for the CGC: (i) Greenschist facies (pre-S $\mathrm{S}_{2}$ ): $\mathrm{Ep}_{1}+\mathrm{Pl}_{1}+$ $\mathrm{Ttn}_{1}+\mathrm{Amp}_{1}+\mathrm{Wm}_{1}+\mathrm{Qtz}$; (ii) Blueschist facies (syn- to post-S $\mathrm{S}_{2}$ : $\mathrm{Amp}_{2}+\mathrm{Chl}+\mathrm{Ep}_{2}+\mathrm{Pl}_{2}+\mathrm{Mt}+$ $\mathrm{Wm} 1+\operatorname{Rt}\left(\mathrm{Ttn}_{2}\right)$

\section{4.c. Ophiolitic Complex (meta-basalts)}

As described in Section 3.c, meta-basalts crop out in the southern area of the AMC in tectonic contact with serpentinized ultramafic rocks (Figs 1, 2). As observed in outcrops, where the primary pillow structures are completely preserved, the meta-basalts also show little deformation at the microscale. The complete mineralogy is defined by: amphibole, plagioclase, chlorite, epidote, white mica, magnetite, apatite and $\mathrm{Fe}-\mathrm{Ti}$ oxides.

Meta-basalts are fine grained with a very poorly defined foliation $\left(\mathrm{S}_{1}\right)$ made by the SPO of $\mathrm{Pl}_{1}+\mathrm{Chl}+\mathrm{Amp}_{1}+\mathrm{Ep}_{1}+\mathrm{Ttn}_{1}$, with $\mathrm{Amp}_{1}$ displaying an actinolitic composition. This weak foliation is overprinted by the static growth of prismatic Na-rich blue amphibole $\left(\mathrm{Amp}_{2}\right)$ and aggregates of titanite crystals $\left(\mathrm{Ttn}_{2}\right)$ that show no evidence of deformation. Besides their occurrence as relatively large (up to $2 \mathrm{~mm}$ ) prismatic crystals (Fig. 5), Amp 2 has also been observed to grow as thin coronae around $\mathrm{Chl}+\mathrm{Amp}_{1}$ sites (Fig. 5). Based on microstructural analysis, the meta-pillow basalts show a prograde metamorphic 
evolution, with a greenschist-facies foliation overprinted by the static growth of a HP-LT blueschist-facies phase assemblage as summarized below:

(i) Greenschist (syn- $\left.\mathrm{S}_{1}\right): \quad \mathrm{Amp}_{1}+\mathrm{Pl}_{1}+\mathrm{Chl}+$ $\mathrm{Wm}_{1}+\mathrm{Ep}_{1}+\mathrm{Ttn}_{1}+\mathrm{Qtz}$; (ii) Blueschist (post-S $_{1}$ : $\mathrm{Amp}_{2}+\mathrm{Ttn}_{2}+\mathrm{Wm}_{2}$.

\section{Mineral chemistry and $\boldsymbol{P}-\boldsymbol{T}-\mathrm{d}-\boldsymbol{t}$ paths}

\section{5.a. Methods}

Textural and mineralogical features were inspected on polished thin-sections using back-scattered electron (BSE) and secondary electron images and characterized by electron microprobe analyses. Analyses were performed at the Earth Science Department of the University of Milano on a Jeol JXA 8200 Superprobe equipped with five wavelength-dispersive spectrometers (WDS). Analyses were conducted with a $c$. $1 \mu \mathrm{m}$ beam diameter at conditions of $15 \mathrm{kV}$ and $5 \mathrm{nA}$ of probe current. Natural silicates and oxides were used as standards. All standards were calibrated within $0.5 \%$ at one standard deviation. Raw data were corrected using a Phi-Rho-Z quantitative analysis program. Major elements and recalculated structural formulae are reported in Tables 2, 3 and 4.

Whole-rock analysis for major elements, used for the isochemical $P-T$ pseudosection (see below), was determined at the ACME Analytical Laboratories Ltd in Vancouver (Canada). Total abundances of the major oxides were obtained by ICP-ES (inductively coupled plasma emission spectroscopy) following a $\mathrm{LiBO}_{2}$ fusion and dilute nitric digestion for major oxides. Analytical errors are within $2 \%$.

The $\mathrm{FeO}$ weight $\%$ on total $\mathrm{FeO}^{*}$ has been determined for the AK14 sample by titration following the Wilson method (Wilson, 1955; Yokoyama \& Nakamura, 2002 and references therein) at the Department of Earth and Environmental Sciences, University of Milano Bicocca.

\section{5.b. Results}

\section{5.b.1. Morghab Complex}

Porphyroblastic epidote and plagioclase preserve a relict paragenesis composed by $\mathrm{Amp}_{1}+\mathrm{Wm}_{1}+\mathrm{Ttn}_{1}+\mathrm{Ep}_{1}+\mathrm{Pl}_{1}+\mathrm{Qtz}$ (fig. $12 \mathrm{~b}$ in Zanchi et al. 2015). Micron-sized $A \mathrm{mp}_{1}$ is an alkali-amphibole $(\mathrm{NaB}>1.50)$ and corresponds to a glaucophane (Table 2), while $\mathrm{Amp}_{2}$ along the main foliation is calcic to sodic-calcic $(\mathrm{Ca}+\mathrm{Na}) \mathrm{B}>1$, $0.5<\mathrm{NaB}<1.5$ and variable $\mathrm{CaB}$. This mainly corresponds to actinolite and magnesiokatophorite and shows a slight zoning from core to rim, with a decrease in $\mathrm{CaO}$, complementary to a slight increase in $\mathrm{Na}_{2} \mathrm{O}$ (Table 2; Fig. 6d). In addition epidote shows a strong compositional variation. $\mathrm{Ep}_{1}$ included in albite porphyroblasts show higher $\mathrm{FeO}^{*}$ contents with respect to the cores of $\mathrm{Ep}_{2}$, which in turn is characterized by a compositional zoning with the rim enriched in
$\mathrm{FeO}^{*}$ with respect to the core (Table 2). This prograde zoning is likely related to the core-to-rim increase in $\mathrm{Na}_{2} \mathrm{O}$ recorded by $\mathrm{Amp}_{2}$. Both white micas, Wm1 included in porphyroblastic albite and $\mathrm{Wm}_{2}$ along the $\mathrm{S}_{2}$ foliation, show a moderate celadonitic content, in the range of 3.35-3.50 atoms per formula unit (apfu) (Table 2). Finally, plagioclase is mainly albite and does not show any anorthite substitution in both generations.

\section{5.b.2. Chah Gorbeh Complex}

Different from the Morghab mafic rocks, these samples show a prograde evolution from greenschist-facies to a blueschist-facies overprint, as recorded also by a different mineral composition. The main foliation is in fact characterized by rare actinolitic amphibole and abundant alkali-amphiboles $(\mathrm{NaB}>1.50)$ showing homogeneous composition with the highest $\mathrm{Na}$ contents (up to 2.03 apfu, Table 3). They are classified as ferroglaucophane. In a Na v. Ca (Fig. 6a, b) and $(\mathrm{Na}+\mathrm{K})$ v. $\mathrm{Al}^{\mathrm{IV}}{ }^{\mathrm{IV}}$ diagram (Fig. $6 \mathrm{~d}$, e) they show a trend similar to that of amphiboles from the MC with the difference that this is prograde. $\mathrm{Na}$ and $\mathrm{Na}+\mathrm{K}$ become enriched with respect to $\mathrm{Ca}$ and $\mathrm{Al}^{(\mathrm{IV})}$, respectively, from $A \mathrm{Amp}_{1}$ to $\mathrm{Amp}_{2}$ as indicated by the grey arrow. Glaucophane is characterized by inclusion-rich rims. Such inclusions consist of the same mineral phases as those crystallized along the main foliation (Chl, $\mathrm{Ab}$, Wm, Ep and rutile with Ttn coronae). In some samples, epidote along the $S_{2}$ foliation also shows a poikiloblastic texture and includes quartz, albite and titanite. Such epidotes are enriched in $\mathrm{FeO}^{*}$, in agreement with equilibration with glaucophane (Table 3) and do not show the strong iron variability as recorded by epidotes of the mafic rocks from the MC. Both white micas, $\mathrm{Wm}_{1}$ included in glaucophane rims and porphyroblastic albite, and $\mathrm{Wm}_{2}$ along the $\mathrm{S}_{2}$ foliation, show a moderate celadonitic content, in the range of 3.30-3.40 apfu (Table 3). Again, plagioclase is mainly albite and does not show any anorthite substitution.

\section{5.b.3 Ophiolitic Complex (meta-basalts)}

Meta-basalts from the OC show a very weak foliation made of $\mathrm{Pl}_{1}+\mathrm{Chl}+\mathrm{Amp}_{1}+\mathrm{Ep}_{1}+\mathrm{Ttn}_{1}$, with $\mathrm{Amp}_{1}$ displaying an actinolitic composition, overprinted by the static growth of $\mathrm{Amp}_{2}$ and aggregates of $\mathrm{Ttn}_{2}$. Amp $\mathrm{Am}_{2}$ occur both as fine-grained aggregates and as millimetre-sized prismatic crystals. As shown in Table 4, fine-grained $\mathrm{Amp}_{2}$ has a variable composition, locally between alkali to sodic-calcic amphiboles (NaB from 1.29 to $1.69 \mathrm{apfu}$ ). Coarse $\mathrm{Amp}_{2}$ is instead homogeneous in composition and corresponds to a magnesioriebeckite. It shows slightly lower $\mathrm{Na}$ content with respect to alkali-amphibole occurring in the Chah Gorbeh mafic rocks (Table 4; Fig. 6c, f). Some coarse white micas appear in microstructural equilibrium with $\mathrm{Amp}_{2}$ and are characterized by a higher celadonitic substitution, up to 3.55 (Table 4), with 
Table 2. Average composition of major elements (ox. wt \%) and of recalculated structural formulae of minerals composing mafic rocks from the Morghab Complex (numbers in brackets are standard deviations)

\begin{tabular}{|c|c|c|c|c|c|c|c|c|c|c|c|c|c|c|c|c|c|c|c|c|c|}
\hline \multirow{5}{*}{$\begin{array}{l}\text { Sample Mineral } \\
\text { Microstructural } \\
\text { position } \\
\text { No. analyses }\end{array}$} & \multicolumn{6}{|c|}{ Amphibole } & \multicolumn{5}{|c|}{ Epidote } & \multirow{2}{*}{\multicolumn{2}{|c|}{$\begin{array}{l}\text { Titanite } \\
\text { AK14A }\end{array}$}} & \multicolumn{4}{|c|}{ Plagioclase } & \multicolumn{3}{|c|}{ Phengite } & \multirow{5}{*}{$\begin{array}{l}\text { AK19B } \\
\text { Chlorite } \\
\text { Chl }\end{array}$} \\
\hline & \multicolumn{3}{|c|}{ AK14A } & \multicolumn{3}{|c|}{ AK19B } & \multicolumn{3}{|c|}{ AK14A } & \multicolumn{2}{|c|}{ AK19B } & & & \multicolumn{3}{|c|}{ AK14A } & AK19B & \multicolumn{2}{|c|}{ AK14A } & \multirow{2}{*}{$\begin{array}{l}\text { AK19B } \\
\\
\mathrm{Wm}_{1} \\
\mathrm{Inc} \text { in }\end{array}$} & \\
\hline & $\begin{array}{l}\text { Amp } 1 \\
\text { Inc in }\end{array}$ & $\mathrm{Amp}_{2}$ & $\mathrm{Amp}_{2}$ & $\begin{array}{l}\text { Amp }_{1} \\
\text { Inc in }\end{array}$ & $\mathrm{Amp}_{2}$ & $\mathrm{Amp}_{2}$ & $\begin{array}{l}\mathrm{Ep}_{1} \\
\text { Inc in }\end{array}$ & $\mathrm{Ep}_{2}$ & $\mathrm{Ep}_{2}$ & $\mathrm{Ep}_{2}$ & $\mathrm{Ep}_{2}$ & $\begin{array}{l}\mathrm{Ttn}_{1} \\
\text { Inc in }\end{array}$ & & $\begin{array}{l}\mathrm{Ab} \\
\text { Porph }\end{array}$ & $\begin{array}{c}\mathrm{Ab} \\
\text { Porph }\end{array}$ & & $\mathrm{Ab}$ & $\begin{array}{l}\mathrm{Wm}_{1} \\
\mathrm{Inc} \text { in }\end{array}$ & & & \\
\hline & & Core & Rim & $\mathrm{Ep}_{2}$ & Core & $\operatorname{Rim}$ & $\mathrm{Ab}$ & Core & Rim & Core & Rim & Ep2 & $\mathrm{Ttn}_{2}$ & Core & Rim & $\mathrm{Ab}_{2}$ & Porph & $\mathrm{Ab}$ & $\mathrm{Wm}_{2}$ & $\mathrm{Ab}$ & \\
\hline & 9 & 5 & 5 & 7 & 12 & 14 & 2 & 4 & 6 & 4 & 4 & 2 & 4 & 1 & 1 & 1 & 2 & 3 & 8 & 3 & \\
\hline $\mathrm{SiO}_{2}$ & $\begin{array}{l}55.79 \\
(0.78)\end{array}$ & $\begin{array}{l}51.74 \\
(1.11)\end{array}$ & $\begin{array}{l}50.83 \\
(0.99)\end{array}$ & $\begin{array}{l}55.85 \\
(0.48)\end{array}$ & $\begin{array}{l}51.40 \\
(1.81)\end{array}$ & $\begin{array}{l}49.44 \\
(1.06)\end{array}$ & $\begin{array}{l}37.96 \\
(0.35)\end{array}$ & $\begin{array}{l}38.23 \\
(0.25)\end{array}$ & $\begin{array}{l}37.49 \\
(0.26)\end{array}$ & $\begin{array}{l}37.92 \\
(0.19)\end{array}$ & $\begin{array}{l}37.18 \\
(0.44)\end{array}$ & $\begin{array}{l}30.78 \\
(0.37)\end{array}$ & $\begin{array}{l}30.34 \\
(0.08)\end{array}$ & 69.21 & 68.63 & 69.33 & $\begin{array}{l}68.54 \\
(0.52)\end{array}$ & $\begin{array}{l}51.41 \\
(0.78)\end{array}$ & $\begin{array}{l}52.29 \\
(1.99)\end{array}$ & $\begin{array}{l}51.29 \\
(1.35)\end{array}$ & $\begin{array}{l}26.54 \\
(0.52)\end{array}$ \\
\hline $\mathrm{TiO}_{2}$ & $\begin{array}{c}0.03 \\
(0.03)\end{array}$ & $\begin{array}{c}0.50 \\
(0.96)\end{array}$ & $\begin{array}{c}0.06 \\
(0.02)\end{array}$ & $\begin{array}{c}0.03 \\
(0.02)\end{array}$ & $\begin{array}{c}0.07 \\
(0.05)\end{array}$ & $\begin{array}{c}0.12 \\
(0.05)\end{array}$ & $\begin{array}{c}0.06 \\
(0.00)\end{array}$ & $\begin{array}{c}0.05 \\
(0.02)\end{array}$ & $\begin{array}{c}0.04 \\
(0.03)\end{array}$ & $\begin{array}{c}0.06 \\
(0.03)\end{array}$ & $\begin{array}{c}0.04 \\
(0.04)\end{array}$ & $\begin{array}{l}35.36 \\
(2.59)\end{array}$ & $\begin{array}{l}38.03 \\
(0.29)\end{array}$ & 0.01 & 0.00 & 0.00 & $\begin{array}{c}0.00 \\
(0.00)\end{array}$ & $\begin{array}{c}0.07 \\
(0.02)\end{array}$ & $\begin{array}{c}0.14 \\
(0.11)\end{array}$ & $\begin{array}{c}0.07 \\
(0.03)\end{array}$ & $\begin{array}{c}0.08 \\
(0.06)\end{array}$ \\
\hline $\mathrm{A}_{12} \mathrm{O}_{3}$ & $\begin{array}{c}8.58 \\
(1.10)\end{array}$ & $\begin{array}{c}4.02 \\
(1.46)\end{array}$ & $\begin{array}{c}4.55 \\
(0.93)\end{array}$ & $\begin{array}{c}7.99 \\
(0.89)\end{array}$ & $\begin{array}{c}3.75 \\
(1.47)\end{array}$ & $\begin{array}{c}6.44 \\
(0.77)\end{array}$ & $\begin{array}{l}24.47 \\
(2.54)\end{array}$ & $\begin{array}{l}26.55 \\
(0.27)\end{array}$ & $\begin{array}{l}22.63 \\
(0.24)\end{array}$ & $\begin{array}{l}25.23 \\
(0.73)\end{array}$ & $\begin{array}{l}23.20 \\
(1.31)\end{array}$ & $\begin{array}{c}1.88 \\
(1.33)\end{array}$ & $\begin{array}{c}1.01 \\
(0.20)\end{array}$ & 19.88 & 19.76 & 20.00 & $\begin{array}{l}19.74 \\
(0.21)\end{array}$ & $\begin{array}{l}28.44 \\
(1.84)\end{array}$ & $\begin{array}{l}25.65 \\
(1.55)\end{array}$ & $\begin{array}{l}30.47 \\
(2.29)\end{array}$ & $\begin{array}{l}19.62 \\
(0.25)\end{array}$ \\
\hline $\mathrm{Cr}_{2} \mathrm{O}_{3}$ & $\begin{array}{c}0.01 \\
(0.01)\end{array}$ & $\begin{array}{c}0.01 \\
(0.02)\end{array}$ & $\begin{array}{c}0.02 \\
(0.03)\end{array}$ & $\begin{array}{c}0.00 \\
(0.01)\end{array}$ & $\begin{array}{c}0.03 \\
(0.03)\end{array}$ & $\begin{array}{c}0.01 \\
(0.02)\end{array}$ & $\begin{array}{c}0.03 \\
(0.04)\end{array}$ & $\begin{array}{c}0.02 \\
(0.03)\end{array}$ & $\begin{array}{c}0.00 \\
(0.00)\end{array}$ & $\begin{array}{c}0.02 \\
(0.03)\end{array}$ & $\begin{array}{c}0.03 \\
(0.03)\end{array}$ & $\begin{array}{c}0.06 \\
(0.01)\end{array}$ & $\begin{array}{c}0.01 \\
(0.02)\end{array}$ & 0.01 & 0.02 & 0.02 & $\begin{array}{c}0.02 \\
(0.03)\end{array}$ & $\begin{array}{c}0.02 \\
(0.04)\end{array}$ & $\begin{array}{c}0.01 \\
(0.01)\end{array}$ & $\begin{array}{c}0.03 \\
(0.05)\end{array}$ & $\begin{array}{c}0.00 \\
(0.00)\end{array}$ \\
\hline $\mathrm{FeO}^{*}$ & $\begin{array}{l}17.82 \\
(0.52)\end{array}$ & $\begin{array}{l}17.06 \\
(0.94)\end{array}$ & $\begin{array}{l}19.41 \\
(1.17)\end{array}$ & $\begin{array}{l}16.91 \\
(0.76)\end{array}$ & $\begin{array}{l}16.17 \\
(1.82)\end{array}$ & $\begin{array}{l}18.44 \\
(0.72)\end{array}$ & $\begin{array}{l}10.68 \\
(3.10)\end{array}$ & $\begin{array}{c}8.61 \\
(0.25)\end{array}$ & $\begin{array}{l}13.25 \\
(0.37)\end{array}$ & $\begin{array}{l}10.26 \\
(0.96)\end{array}$ & $\begin{array}{l}12.69 \\
(1.83)\end{array}$ & $\begin{array}{c}1.17 \\
(0.63)\end{array}$ & $\begin{array}{c}0.47 \\
(0.15)\end{array}$ & 0.01 & 0.09 & 0.14 & $\begin{array}{c}0.09 \\
(0.11)\end{array}$ & $\begin{array}{c}4.04 \\
(0.26)\end{array}$ & $\begin{array}{c}5.20 \\
(1.67)\end{array}$ & $\begin{array}{c}3.02 \\
(0.39)\end{array}$ & $\begin{array}{l}25.92 \\
(0.31)\end{array}$ \\
\hline $\mathrm{MgO}$ & $\begin{array}{c}6.65 \\
(0.36)\end{array}$ & $\begin{array}{l}11.34 \\
(0.98)\end{array}$ & $\begin{array}{l}10.12 \\
(0.87)\end{array}$ & $\begin{array}{c}7.91 \\
(0.27)\end{array}$ & $\begin{array}{l}12.73 \\
(1.41)\end{array}$ & $\begin{array}{l}10.44 \\
(0.63)\end{array}$ & $\begin{array}{c}0.01 \\
(0.02)\end{array}$ & $\begin{array}{c}0.02 \\
(0.01)\end{array}$ & $\begin{array}{c}0.01 \\
(0.01)\end{array}$ & $\begin{array}{c}0.01 \\
(0.01)\end{array}$ & $\begin{array}{c}0.03 \\
(0.01)\end{array}$ & $\begin{array}{c}0.00 \\
(0.00)\end{array}$ & $\begin{array}{c}0.01 \\
(0.01)\end{array}$ & 0.00 & 0.00 & 0.01 & $\begin{array}{c}0.01 \\
(0.00)\end{array}$ & $\begin{array}{c}2.67 \\
(1.50)\end{array}$ & $\begin{array}{c}3.10 \\
(0.39)\end{array}$ & $\begin{array}{c}2.00 \\
(0.63)\end{array}$ & $\begin{array}{l}15.79 \\
(0.30)\end{array}$ \\
\hline $\mathrm{MnO}$ & $\begin{array}{c}0.18 \\
(0.05)\end{array}$ & $\begin{array}{c}0.28 \\
(0.02)\end{array}$ & $\begin{array}{c}0.32 \\
(0.04)\end{array}$ & $\begin{array}{c}0.17 \\
(0.05)\end{array}$ & $\begin{array}{c}0.35 \\
(0.06)\end{array}$ & $\begin{array}{c}0.36 \\
(0.03)\end{array}$ & $\begin{array}{c}0.17 \\
(0.09)\end{array}$ & $\begin{array}{c}0.14 \\
(0.04)\end{array}$ & $\begin{array}{l}0.21 \\
(0.04)\end{array}$ & $\begin{array}{c}0.23 \\
(0.08)\end{array}$ & $\begin{array}{c}0.20 \\
(0.08)\end{array}$ & $\begin{array}{c}0.03 \\
(0.02)\end{array}$ & $\begin{array}{c}0.03 \\
(0.03)\end{array}$ & 0.02 & 0.00 & 0.00 & $\begin{array}{c}0.00 \\
(0.00)\end{array}$ & $\begin{array}{c}0.02 \\
(0.02)\end{array}$ & $\begin{array}{c}0.04 \\
(0.04)\end{array}$ & $\begin{array}{c}0.02 \\
(0.03)\end{array}$ & $\begin{array}{c}0.42 \\
(0.09)\end{array}$ \\
\hline $\mathrm{CaO}$ & $\begin{array}{c}1.66 \\
(0.88)\end{array}$ & $\begin{array}{l}10.01 \\
(0.76)\end{array}$ & $\begin{array}{c}8.73 \\
(0.66)\end{array}$ & $\begin{array}{c}1.00 \\
(0.32)\end{array}$ & $\begin{array}{l}10.32 \\
(0.89)\end{array}$ & $\begin{array}{c}7.90 \\
(0.41)\end{array}$ & $\begin{array}{l}23.03 \\
(0.44)\end{array}$ & $\begin{array}{l}23.37 \\
(0.09)\end{array}$ & $\begin{array}{l}22.56 \\
(0.11)\end{array}$ & $\begin{array}{l}23.16 \\
(0.24)\end{array}$ & $\begin{array}{l}22.75 \\
(0.13)\end{array}$ & $\begin{array}{l}28.02 \\
(0.16)\end{array}$ & $\begin{array}{l}28.19 \\
(0.17)\end{array}$ & 0.06 & 0.05 & 0.08 & $\begin{array}{c}0.05 \\
(0.01)\end{array}$ & $\begin{array}{c}0.09 \\
(0.09)\end{array}$ & $\begin{array}{c}0.07 \\
(0.04)\end{array}$ & $\begin{array}{c}0.01 \\
(0.01)\end{array}$ & $\begin{array}{c}0.04 \\
(0.01)\end{array}$ \\
\hline $\mathrm{Na}_{2} \mathrm{O}$ & $\begin{array}{c}6.70 \\
(0.28)\end{array}$ & $\begin{array}{c}1.89 \\
(0.44)\end{array}$ & $\begin{array}{c}2.56 \\
(0.50)\end{array}$ & $\begin{array}{c}6.77 \\
(0.17)\end{array}$ & $\begin{array}{c}1.50 \\
(0.57)\end{array}$ & $\begin{array}{c}3.17 \\
(0.26)\end{array}$ & $\begin{array}{c}0.01 \\
(0.01)\end{array}$ & $\begin{array}{c}0.00 \\
(0.00)\end{array}$ & $\begin{array}{c}0.01 \\
(0.01)\end{array}$ & $\begin{array}{c}0.01 \\
(0.00)\end{array}$ & $\begin{array}{c}0.01 \\
(0.02)\end{array}$ & $\begin{array}{c}0.01 \\
(0.02)\end{array}$ & $\begin{array}{c}0.02 \\
(0.02)\end{array}$ & 11.87 & 11.96 & 11.50 & $\begin{array}{l}11.47 \\
(0.04)\end{array}$ & $\begin{array}{c}0.28 \\
(0.05)\end{array}$ & $\begin{array}{c}0.15 \\
(0.05)\end{array}$ & $\begin{array}{c}0.38 \\
(0.10)\end{array}$ & $\begin{array}{c}0.01 \\
(0.01)\end{array}$ \\
\hline $\mathrm{K}_{2} \mathrm{O}$ & $\begin{array}{c}0.02 \\
(0.02)\end{array}$ & $\begin{array}{c}0.17 \\
(0.05)\end{array}$ & $\begin{array}{c}0.21 \\
(0.04)\end{array}$ & $\begin{array}{c}0.01 \\
(0.01)\end{array}$ & $\begin{array}{c}0.15 \\
(0.07)\end{array}$ & $\begin{array}{c}0.22 \\
(0.04)\end{array}$ & $\begin{array}{c}0.00 \\
(0.00)\end{array}$ & $\begin{array}{c}0.00 \\
(0.00)\end{array}$ & $\begin{array}{c}0.00 \\
(0.01)\end{array}$ & $\begin{array}{c}0.00 \\
(0.00)\end{array}$ & $\begin{array}{c}0.00 \\
(0.00)\end{array}$ & $\begin{array}{c}0.01 \\
(0.01)\end{array}$ & $\begin{array}{c}0.02 \\
(0.01)\end{array}$ & 0.04 & 0.05 & 0.04 & $\begin{array}{c}0.05 \\
(0.00)\end{array}$ & $\begin{array}{c}9.43 \\
(0.23)\end{array}$ & $\begin{array}{c}9.74 \\
(0.21)\end{array}$ & $\begin{array}{c}9.78 \\
(0.16)\end{array}$ & $\begin{array}{c}0.01 \\
(0.01)\end{array}$ \\
\hline Total & $\begin{array}{l}97.45 \\
(0.43)\end{array}$ & $\begin{array}{l}97.01 \\
(0.45)\end{array}$ & $\begin{array}{l}96.81 \\
(0.41)\end{array}$ & $\begin{array}{l}97.36 \\
(1.29)\end{array}$ & $\begin{array}{l}96.56 \\
(0.77)\end{array}$ & $\begin{array}{l}96.80 \\
(0.79)\end{array}$ & $\begin{array}{l}96.42 \\
(0.21)\end{array}$ & $\begin{array}{l}96.99 \\
(0.26)\end{array}$ & $\begin{array}{l}96.21 \\
(0.25)\end{array}$ & $\begin{array}{l}96.90 \\
(0.13)\end{array}$ & $\begin{array}{l}96.13 \\
(0.32)\end{array}$ & $\begin{array}{l}97.32 \\
(0.14)\end{array}$ & $\begin{array}{l}98.14 \\
(0.38)\end{array}$ & 101.11 & 100.56 & 101.12 & $\begin{array}{l}99.96 \\
(0.26)\end{array}$ & $\begin{array}{l}96.47 \\
(0.95)\end{array}$ & $\begin{array}{l}96.40 \\
(1.30)\end{array}$ & $\begin{array}{l}97.06 \\
(0.18)\end{array}$ & $\begin{array}{l}88.40 \\
(0.23)\end{array}$ \\
\hline $\mathrm{Si}$ & $\begin{array}{c}7.94 \\
(0.11)\end{array}$ & $\begin{array}{l}7.59 \\
(0.15)\end{array}$ & $\begin{array}{c}7.54 \\
(0.11)\end{array}$ & $\begin{array}{c}7.98 \\
(0.06)\end{array}$ & $\begin{array}{c}7.56 \\
(0.19)\end{array}$ & $\begin{array}{c}7.34 \\
(0.12)\end{array}$ & $\begin{array}{c}3.02 \\
(0.01)\end{array}$ & $\begin{array}{c}3.00 \\
(0.01)\end{array}$ & $\begin{array}{l}3.01 \\
(0.02)\end{array}$ & $\begin{array}{c}2.99 \\
(0.02)\end{array}$ & $\begin{array}{l}2.98 \\
(0.02)\end{array}$ & $\begin{array}{c}1.02 \\
(0.01)\end{array}$ & $\begin{array}{c}1.00 \\
(0.00)\end{array}$ & 2.99 & 2.98 & 3.00 & $\begin{array}{l}3.00 \\
(0.02)\end{array}$ & $\begin{array}{c}3.39 \\
(0.03)\end{array}$ & $\begin{array}{c}3.47 \\
(0.07)\end{array}$ & $\begin{array}{l}3.34 \\
(0.09)\end{array}$ & $\begin{array}{c}2.79 \\
(0.05)\end{array}$ \\
\hline $\mathrm{Ti}$ & $\begin{array}{c}0.00 \\
(0.00)\end{array}$ & $\begin{array}{c}0.05 \\
(0.11)\end{array}$ & $\begin{array}{c}0.01 \\
(0.00)\end{array}$ & $\begin{array}{c}0.00 \\
(0.00)\end{array}$ & $\begin{array}{c}0.01 \\
(0.01)\end{array}$ & $\begin{array}{c}0.01 \\
(0.01)\end{array}$ & $\begin{array}{c}0.00 \\
(0.00)\end{array}$ & $\begin{array}{c}0.00 \\
(0.00)\end{array}$ & $\begin{array}{c}0.00 \\
(0.00)\end{array}$ & $\begin{array}{c}0.00 \\
(0.00)\end{array}$ & $\begin{array}{c}0.00 \\
(0.00)\end{array}$ & $\begin{array}{c}0.88 \\
(0.07)\end{array}$ & $\begin{array}{c}0.94 \\
(0.01)\end{array}$ & 0.00 & 0.00 & 0.00 & $\begin{array}{c}0.00 \\
(0.00)\end{array}$ & $\begin{array}{c}0.00 \\
(0.00)\end{array}$ & $\begin{array}{c}0.01 \\
(0.01)\end{array}$ & $\begin{array}{c}0.00 \\
(0.00)\end{array}$ & $\begin{array}{c}0.01 \\
(0.01)\end{array}$ \\
\hline $\mathrm{Al}$ & $\begin{array}{c}1.44 \\
(0.18)\end{array}$ & $\begin{array}{c}0.70 \\
(0.25)\end{array}$ & $\begin{array}{c}0.80 \\
(0.17)\end{array}$ & $\begin{array}{c}1.35 \\
(0.15)\end{array}$ & $\begin{array}{c}0.65 \\
(0.26)\end{array}$ & $\begin{array}{c}1.13 \\
(0.14)\end{array}$ & $\begin{array}{c}2.29 \\
(0.21)\end{array}$ & $\begin{array}{c}2.46 \\
(0.02)\end{array}$ & $\begin{array}{c}2.14 \\
(0.02)\end{array}$ & $\begin{array}{c}2.35 \\
(0.06)\end{array}$ & $\begin{array}{c}2.19 \\
(0.11)\end{array}$ & $\begin{array}{c}0.07 \\
(0.05)\end{array}$ & $\begin{array}{c}0.04 \\
(0.01)\end{array}$ & 1.01 & 1.01 & 1.02 & $\begin{array}{c}1.02 \\
(0.01)\end{array}$ & $\begin{array}{c}2.21 \\
(0.14)\end{array}$ & $\begin{array}{c}2.01 \\
(0.11)\end{array}$ & $\begin{array}{l}2.34 \\
(0.17)\end{array}$ & $\begin{array}{c}2.43 \\
(0.04)\end{array}$ \\
\hline $\mathrm{Cr}$ & $\begin{array}{c}0.00 \\
(0.00)\end{array}$ & $\begin{array}{c}0.00 \\
(0.00)\end{array}$ & $\begin{array}{c}0.00 \\
(0.00)\end{array}$ & $\begin{array}{c}0.00 \\
(0.00)\end{array}$ & $\begin{array}{c}0.00 \\
(0.00)\end{array}$ & $\begin{array}{c}0.00 \\
(0.00)\end{array}$ & $\begin{array}{c}0.00 \\
(0.00)\end{array}$ & $\begin{array}{c}0.00 \\
(0.00)\end{array}$ & $\begin{array}{c}0.00 \\
(0.00)\end{array}$ & $\begin{array}{c}0.00 \\
(0.00)\end{array}$ & $\begin{array}{c}0.00 \\
(0.00)\end{array}$ & $\begin{array}{c}0.00 \\
(0.00)\end{array}$ & $\begin{array}{c}0.00 \\
(0.00)\end{array}$ & 0.00 & 0.00 & 0.00 & $\begin{array}{c}0.00 \\
(0.00)\end{array}$ & $\begin{array}{c}0.00 \\
(0.00)\end{array}$ & $\begin{array}{c}0.00 \\
(0.00)\end{array}$ & $\begin{array}{c}0.00 \\
(0.00)\end{array}$ & $\begin{array}{c}0.00 \\
(0.00)\end{array}$ \\
\hline $\mathrm{Fe}^{*}$ & $\begin{array}{c}2.12 \\
(0.07)\end{array}$ & $\begin{array}{c}2.09 \\
(0.12)\end{array}$ & $\begin{array}{c}2.41 \\
(0.15)\end{array}$ & $\begin{array}{c}2.02 \\
(0.09)\end{array}$ & $\begin{array}{c}1.99 \\
(0.24)\end{array}$ & $\begin{array}{c}2.29 \\
(0.10)\end{array}$ & $\begin{array}{c}0.71 \\
(0.21)\end{array}$ & $\begin{array}{c}0.56 \\
(0.02)\end{array}$ & $\begin{array}{c}0.89 \\
(0.02)\end{array}$ & $\begin{array}{c}0.68 \\
(0.07)\end{array}$ & $\begin{array}{c}0.85 \\
(0.13)\end{array}$ & $\begin{array}{c}0.03 \\
(0.02)\end{array}$ & $\begin{array}{c}0.01 \\
(0.00)\end{array}$ & 0.00 & 0.00 & 0.00 & $\begin{array}{c}0.00 \\
(0.00)\end{array}$ & $\begin{array}{c}0.22 \\
(0.02)\end{array}$ & $\begin{array}{c}0.29 \\
(0.10)\end{array}$ & $\begin{array}{c}0.16 \\
(0.02)\end{array}$ & $\begin{array}{c}2.28 \\
(0.04)\end{array}$ \\
\hline $\mathrm{Mg}$ & $\begin{array}{c}1.41 \\
(0.08)\end{array}$ & $\begin{array}{c}2.48 \\
(0.20)\end{array}$ & $\begin{array}{c}2.24 \\
(0.18)\end{array}$ & $\begin{array}{c}1.68 \\
(0.06)\end{array}$ & $\begin{array}{c}2.79 \\
(0.28)\end{array}$ & $\begin{array}{c}2.31 \\
(0.13)\end{array}$ & $\begin{array}{c}0.00 \\
(0.00)\end{array}$ & $\begin{array}{c}0.00 \\
(0.00)\end{array}$ & $\begin{array}{c}0.00 \\
(0.00)\end{array}$ & $\begin{array}{c}0.00 \\
(0.00)\end{array}$ & $\begin{array}{c}0.00 \\
(0.00)\end{array}$ & $\begin{array}{c}0.00 \\
(0.00)\end{array}$ & $\begin{array}{c}0.00 \\
(0.00)\end{array}$ & 0.00 & 0.00 & 0.00 & $\begin{array}{c}0.00 \\
(0.00)\end{array}$ & $\begin{array}{c}0.26 \\
(0.15)\end{array}$ & $\begin{array}{c}0.31 \\
(0.04)\end{array}$ & $\begin{array}{c}0.19 \\
(0.06)\end{array}$ & $\begin{array}{c}2.47 \\
(0.04)\end{array}$ \\
\hline $\mathrm{Mn}$ & $\begin{array}{c}0.02 \\
(0.01)\end{array}$ & $\begin{array}{c}0.03 \\
(0.00)\end{array}$ & $\begin{array}{c}0.04 \\
(0.00)\end{array}$ & $\begin{array}{c}0.02 \\
(0.01)\end{array}$ & $\begin{array}{c}0.04 \\
(0.01)\end{array}$ & $\begin{array}{c}0.05 \\
(0.00)\end{array}$ & $\begin{array}{c}0.01 \\
(0.01)\end{array}$ & $\begin{array}{c}0.01 \\
(0.00)\end{array}$ & $\begin{array}{c}0.01 \\
(0.00)\end{array}$ & $\begin{array}{c}0.02 \\
(0.01)\end{array}$ & $\begin{array}{c}0.01 \\
(0.01)\end{array}$ & $\begin{array}{c}0.00 \\
(0.00)\end{array}$ & $\begin{array}{c}0.00 \\
(0.00)\end{array}$ & 0.00 & 0.00 & 0.00 & $\begin{array}{c}0.00 \\
(0.00)\end{array}$ & $\begin{array}{c}0.00 \\
(0.00)\end{array}$ & $\begin{array}{c}0.00 \\
(0.00)\end{array}$ & $\begin{array}{c}0.00 \\
(0.00)\end{array}$ & $\begin{array}{c}0.04 \\
(0.01)\end{array}$ \\
\hline $\mathrm{Ca}$ & $\begin{array}{c}0.25 \\
(0.13)\end{array}$ & $\begin{array}{c}1.57 \\
(0.11)\end{array}$ & $\begin{array}{c}1.39 \\
(0.10)\end{array}$ & $\begin{array}{c}0.15 \\
(0.05)\end{array}$ & $\begin{array}{c}1.63 \\
(0.13)\end{array}$ & $\begin{array}{c}1.26 \\
(0.07)\end{array}$ & $\begin{array}{c}1.96 \\
(0.01)\end{array}$ & $\begin{array}{c}1.96 \\
(0.01)\end{array}$ & $\begin{array}{c}1.94 \\
(0.01)\end{array}$ & $\begin{array}{c}1.96 \\
(0.01)\end{array}$ & $\begin{array}{c}1.95 \\
(0.00)\end{array}$ & $\begin{array}{c}0.99 \\
(0.00)\end{array}$ & $\begin{array}{c}1.00 \\
(0.00)\end{array}$ & 0.00 & 0.00 & 0.00 & $\begin{array}{c}0.00 \\
(0.00)\end{array}$ & $\begin{array}{c}0.01 \\
(0.01)\end{array}$ & $\begin{array}{c}0.01 \\
(0.00)\end{array}$ & $\begin{array}{c}0.00 \\
(0.00)\end{array}$ & $\begin{array}{c}0.00 \\
(0.00)\end{array}$ \\
\hline $\mathrm{Na}$ & $\begin{array}{c}1.85 \\
(0.07)\end{array}$ & $\begin{array}{c}0.54 \\
(0.13)\end{array}$ & $\begin{array}{c}0.74 \\
(0.15)\end{array}$ & $\begin{array}{c}1.87 \\
(0.05)\end{array}$ & $\begin{array}{c}0.43 \\
(0.17)\end{array}$ & $\begin{array}{c}0.91 \\
(0.08)\end{array}$ & $\begin{array}{c}0.00 \\
(0.00)\end{array}$ & $\begin{array}{c}0.00 \\
(0.00)\end{array}$ & $\begin{array}{c}0.00 \\
(0.00)\end{array}$ & $\begin{array}{c}0.00 \\
(0.00)\end{array}$ & $\begin{array}{c}0.00 \\
(0.00)\end{array}$ & $\begin{array}{c}0.00 \\
(0.00)\end{array}$ & $\begin{array}{c}0.00 \\
(0.00)\end{array}$ & 0.99 & 1.01 & 0.97 & $\begin{array}{c}0.97 \\
(0.00)\end{array}$ & $\begin{array}{c}0.04 \\
(0.01)\end{array}$ & $\begin{array}{c}0.02 \\
(0.01)\end{array}$ & $\begin{array}{c}0.05 \\
(0.01)\end{array}$ & $\begin{array}{c}0.00 \\
(0.00)\end{array}$ \\
\hline K & $\begin{array}{c}0.00 \\
(0.00)\end{array}$ & $\begin{array}{c}0.03 \\
(0.01)\end{array}$ & $\begin{array}{c}0.04 \\
(0.01)\end{array}$ & $\begin{array}{c}0.00 \\
(0.00)\end{array}$ & $\begin{array}{c}0.03 \\
(0.01)\end{array}$ & $\begin{array}{c}0.04 \\
(0.01)\end{array}$ & $\begin{array}{c}0.00 \\
(0.00)\end{array}$ & $\begin{array}{c}0.00 \\
(0.00)\end{array}$ & $\begin{array}{c}0.00 \\
(0.00)\end{array}$ & $\begin{array}{c}0.00 \\
(0.00)\end{array}$ & $\begin{array}{c}0.00 \\
(0.00)\end{array}$ & $\begin{array}{c}0.00 \\
(0.00)\end{array}$ & $\begin{array}{c}0.00 \\
(0.00)\end{array}$ & 0.00 & 0.00 & 0.00 & $\begin{array}{c}0.00 \\
(0.00)\end{array}$ & $\begin{array}{c}0.79 \\
(0.02)\end{array}$ & $\begin{array}{c}0.83 \\
(0.02)\end{array}$ & $\begin{array}{c}0.81 \\
(0.01)\end{array}$ & $\begin{array}{c}0.00 \\
(0.00)\end{array}$ \\
\hline Total & $\begin{array}{l}15.05 \\
(0.02)\end{array}$ & $\begin{array}{l}15.09 \\
(0.08)\end{array}$ & $\begin{array}{c}15.20 \\
(0.09)\end{array}$ & $\begin{array}{l}15.08 \\
(0.04)\end{array}$ & $\begin{array}{l}15.13 \\
(0.11)\end{array}$ & $\begin{array}{l}15.33 \\
(0.05)\end{array}$ & $\begin{array}{c}8.00 \\
(0.00)\end{array}$ & $\begin{array}{c}8.00 \\
(0.00)\end{array}$ & $\begin{array}{c}8.00 \\
(0.00)\end{array}$ & $\begin{array}{c}8.00 \\
(0.00)\end{array}$ & $\begin{array}{c}8.00 \\
(0.00)\end{array}$ & $\begin{array}{c}3.00 \\
(0.00)\end{array}$ & $\begin{array}{c}3.00 \\
(0.00)\end{array}$ & 5.00 & 5.00 & 5.00 & $\begin{array}{c}5.00 \\
(0.00)\end{array}$ & $\begin{array}{c}6.92 \\
(0.03)\end{array}$ & $\begin{array}{c}6.94 \\
(0.05)\end{array}$ & $\begin{array}{c}6.91 \\
(0.00)\end{array}$ & $\begin{array}{l}10.87 \\
(0.00)\end{array}$ \\
\hline
\end{tabular}




\begin{tabular}{|c|c|c|c|c|c|c|c|c|c|c|c|c|c|c|c|c|c|c|c|c|c|c|c|c|c|c|c|}
\hline \multirow[b]{3}{*}{$\begin{array}{l}\text { Sample Mineral } \\
\text { Microstructural } \\
\text { position } \\
\text { No. analyses }\end{array}$} & \multicolumn{7}{|c|}{ Amphibole } & \multicolumn{7}{|c|}{ Epidote } & \multirow{2}{*}{\multicolumn{2}{|c|}{$\begin{array}{c}\text { Titanite } \\
\text { I1309 }\end{array}$}} & \multicolumn{5}{|c|}{ Plagioclase } & \multicolumn{5}{|c|}{ Phengite } & \multirow{3}{*}{$\begin{array}{l}\text { Chlorit } \\
\text { I1309 }\end{array}$} \\
\hline & \multicolumn{3}{|c|}{ I1309 } & \multicolumn{4}{|c|}{ I1311 } & \multicolumn{4}{|c|}{ I1309 } & \multicolumn{3}{|c|}{ I1311 } & & & \multicolumn{3}{|c|}{ I1309 } & \multicolumn{2}{|c|}{ I1311 } & \multicolumn{3}{|c|}{ I1309 } & \multicolumn{2}{|c|}{ I1311 } & \\
\hline & $\begin{array}{c}\text { Amp }_{2} \\
\text { Core } \\
3\end{array}$ & $\begin{array}{c}\mathrm{Amp}_{2} \\
\text { Int } \\
7\end{array}$ & $\begin{array}{c}\mathrm{Amp}_{2} \\
\text { Rim } \\
3\end{array}$ & $\begin{array}{c}\text { Amp }_{1} \\
3\end{array}$ & $\begin{array}{c}\mathrm{Amp}_{2} \\
\text { Core } \\
3\end{array}$ & $\begin{array}{c}\mathrm{Amp}_{2} \\
\text { Int } \\
7\end{array}$ & $\begin{array}{c}\mathrm{Amp}_{2} \\
\text { Rim } \\
5\end{array}$ & $\begin{array}{c}\mathrm{Ep}_{1} \\
\text { Inc in } \\
\mathrm{Ab} \\
3\end{array}$ & $\begin{array}{l}\mathrm{Ep}_{2} \\
\text { Core }\end{array}$ & $\begin{array}{c}\mathrm{Ep}_{2} \\
\text { Int }\end{array}$ & $\begin{array}{l}\mathrm{Ep}_{2} \\
\mathrm{Rim}\end{array}$ & $\begin{array}{l}\mathrm{Ep}_{2} \\
\text { Core }\end{array}$ & $\begin{array}{l}\mathrm{Ep}_{2} \\
\text { Int }\end{array}$ & $\begin{array}{l}\mathrm{Ep}_{2} \\
\text { Rim }\end{array}$ & $\begin{array}{l}\mathrm{Ttn}_{2} \\
\text { Core }\end{array}$ & $\begin{array}{l}\mathrm{Ttn}_{2} \\
\mathrm{Rim}\end{array}$ & $\begin{array}{c}\mathrm{Ab} \\
\text { Porph } \\
\text { Core }\end{array}$ & $\begin{array}{l}\mathrm{Ab} \\
\text { Porph } \\
\text { Int } \\
2\end{array}$ & $\begin{array}{c}\text { Ab } \\
\text { Porph } \\
\text { Rim }\end{array}$ & $\begin{array}{c}\mathrm{Ab} \\
\text { Porph } \\
\text { Core } \\
3\end{array}$ & $\begin{array}{c}\mathrm{Ab} \\
\text { Porph } \\
\text { Rim }\end{array}$ & $\begin{array}{l}\mathrm{Wm}_{1} \\
\mathrm{Inc} \text { in } \\
\mathrm{Amp} 2 \\
5\end{array}$ & $\begin{array}{l}\mathrm{Wm}_{2} \\
\text { Core } \\
7\end{array}$ & $\begin{array}{c}\mathrm{Wm}_{2} \\
\mathrm{Rim} \\
4\end{array}$ & $\begin{array}{l}\mathrm{Wm}_{1} \\
\mathrm{Inc} \text { in } \\
\mathrm{Ab} \\
5\end{array}$ & $\mathrm{Wm}_{2}$ & \\
\hline$b_{2}$ & $\begin{array}{l}55.91 \\
(0.18)\end{array}$ & $\begin{array}{l}55.24 \\
(1.19)\end{array}$ & $\begin{array}{l}56.24 \\
(0.58)\end{array}$ & & $\begin{array}{l}55.29 \\
(1.01)\end{array}$ & $\begin{array}{l}55.75 \\
(0.72)\end{array}$ & $\begin{array}{l}54.95 \\
(1.01)\end{array}$ & $\begin{array}{l}38.07 \\
(0.06)\end{array}$ & $\begin{array}{l}37.21 \\
(0.04)\end{array}$ & $\begin{array}{l}37.79 \\
(0.45)\end{array}$ & $\begin{array}{l}37.92 \\
(0.34)\end{array}$ & 37.83 & 38.26 & 38.27 & 30.91 & 31.05 & $\begin{array}{l}68.86 \\
(0.30)\end{array}$ & $\begin{array}{l}69.08 \\
(0.24)\end{array}$ & $\begin{array}{l}70.09 \\
(1.55)\end{array}$ & $\begin{array}{l}69.29 \\
(0.51)\end{array}$ & $\begin{array}{r}69.33 \\
(0.29)\end{array}$ & $\begin{array}{l}51.69 \\
(0.12)\end{array}$ & $\begin{array}{l}51.42 \\
(0.53)\end{array}$ & $\begin{array}{l}51.21 \\
(0.45)\end{array}$ & $\begin{array}{l}51.45 \\
(1.27)\end{array}$ & $\begin{array}{l}50.78 \\
(0.46)\end{array}$ & $\begin{array}{l}26.10 \\
(0.57)\end{array}$ \\
\hline $\mathrm{iO}_{2}$ & $\begin{array}{c}0.11 \\
(0.04)\end{array}$ & $\begin{array}{c}0.12 \\
(0.09)\end{array}$ & $\begin{array}{c}0.08 \\
(0.03)\end{array}$ & $\begin{array}{c}0.06 \\
(0.05)\end{array}$ & $\begin{array}{c}0.17 \\
(0.08)\end{array}$ & $\begin{array}{c}0.13 \\
(0.06)\end{array}$ & $\begin{array}{c}0.18 \\
(0.06)\end{array}$ & $\begin{array}{c}0.10 \\
(0.03)\end{array}$ & $\begin{array}{c}0.22 \\
(0.14)\end{array}$ & $\begin{array}{c}0.25 \\
(0.04)\end{array}$ & $\begin{array}{c}0.36 \\
(0.38)\end{array}$ & 0.10 & 0.09 & 0.03 & 35.71 & 35.66 & $\begin{array}{c}0.03 \\
(0.04)\end{array}$ & $\begin{array}{c}0.01 \\
(0.01)\end{array}$ & $\begin{array}{c}0.04 \\
(0.04)\end{array}$ & $\begin{array}{c}0.02 \\
(0.00)\end{array}$ & $\begin{array}{c}0.02 \\
(0.02)\end{array}$ & $\begin{array}{c}0.25 \\
(0.08)\end{array}$ & $\begin{array}{c}0.24 \\
(0.07)\end{array}$ & $\begin{array}{c}0.20 \\
(0.06)\end{array}$ & $\begin{array}{c}0.24 \\
(0.09)\end{array}$ & $\begin{array}{c}0.29 \\
(0.07)\end{array}$ & $\begin{array}{c}0.07 \\
(0.03)\end{array}$ \\
\hline${ }_{12} \mathrm{O}_{3}$ & $\begin{array}{c}8.20 \\
(0.35)\end{array}$ & $\begin{array}{c}8.58 \\
(0.49)\end{array}$ & $\begin{array}{c}8.22 \\
(0.49)\end{array}$ & $\begin{array}{c}4.58 \\
(0.63)\end{array}$ & $\begin{array}{c}7.95 \\
(0.71)\end{array}$ & $\begin{array}{c}8.15 \\
(0.55)\end{array}$ & $\begin{array}{c}7.90 \\
(0.43)\end{array}$ & $\begin{array}{l}24.43 \\
(0.30)\end{array}$ & $\begin{array}{l}21.88 \\
(0.78)\end{array}$ & $\begin{array}{l}23.64 \\
(0.69)\end{array}$ & $\begin{array}{l}24.19 \\
(0.43)\end{array}$ & 23.76 & 22.49 & 23.14 & 2.62 & 2.60 & $\begin{array}{l}19.86 \\
(0.38)\end{array}$ & $\begin{array}{l}19.86 \\
(0.01)\end{array}$ & $\begin{array}{l}20.26 \\
(0.23)\end{array}$ & $\begin{array}{l}19.90 \\
(0.15)\end{array}$ & $\begin{array}{l}19.94 \\
(0.15)\end{array}$ & $\begin{array}{l}27.95 \\
(1.97)\end{array}$ & $\begin{array}{l}28.46 \\
(0.53)\end{array}$ & $\begin{array}{l}28.62 \\
(0.27)\end{array}$ & $\begin{array}{l}27.00 \\
(1.01)\end{array}$ & $\begin{array}{l}28.82 \\
(0.59)\end{array}$ & $\begin{array}{l}20.40 \\
(0.44)\end{array}$ \\
\hline $\mathrm{r}_{2} \mathrm{O}_{3}$ & $\begin{array}{c}0.02 \\
(0.02)\end{array}$ & $\begin{array}{c}0.02 \\
(0.01)\end{array}$ & $\begin{array}{c}0.02 \\
(0.04)\end{array}$ & $\begin{array}{c}0.01 \\
(0.00)\end{array}$ & $\begin{array}{c}0.01 \\
(0.02)\end{array}$ & $\begin{array}{c}0.02 \\
(0.03)\end{array}$ & $\begin{array}{c}0.01 \\
(0.01)\end{array}$ & $\begin{array}{c}0.00 \\
(0.00)\end{array}$ & $\begin{array}{c}0.00 \\
(0.00)\end{array}$ & $\begin{array}{c}0.04 \\
(0.03)\end{array}$ & $\begin{array}{c}0.03 \\
(0.03)\end{array}$ & 0.00 & 0.06 & 00 & 0.06 & 0.00 & $\begin{array}{c}0.02 \\
(0.02)\end{array}$ & $\begin{array}{c}0.01 \\
(0.01)\end{array}$ & $\begin{array}{c}0.02 \\
(0.03)\end{array}$ & $\begin{array}{c}0.03 \\
(0.03)\end{array}$ & $\begin{array}{c}0.05 \\
(0.03)\end{array}$ & $\begin{array}{c}0.03 \\
(0.02)\end{array}$ & $\begin{array}{c}0.03 \\
(0.03)\end{array}$ & $\begin{array}{c}0.02 \\
(0.02)\end{array}$ & $\begin{array}{c}0.03 \\
(0.02)\end{array}$ & $\begin{array}{c}0.02 \\
(0.02)\end{array}$ & $\begin{array}{c}0.00 \\
(0.00)\end{array}$ \\
\hline $\mathrm{FeO}^{*}$ & $\begin{array}{l}19.12 \\
(0.55)\end{array}$ & $\begin{array}{l}19.76 \\
(0.69)\end{array}$ & $\begin{array}{l}19.29 \\
(0.26)\end{array}$ & $\begin{array}{l}19.44 \\
(0.33)\end{array}$ & $\begin{array}{l}19.41 \\
(0.24)\end{array}$ & $\begin{array}{l}19.35 \\
(0.55)\end{array}$ & $\begin{array}{l}20.26 \\
(0.86)\end{array}$ & $\begin{array}{l}12.09 \\
(0.20)\end{array}$ & $\begin{array}{l}12.91 \\
(0.52)\end{array}$ & $\begin{array}{l}12.89 \\
(0.39)\end{array}$ & $\begin{array}{l}12.25 \\
(0.36)\end{array}$ & 12.73 & .73 & 13.76 & 0.66 & 0.70 & $\begin{array}{c}0.09 \\
(0.05)\end{array}$ & $\begin{array}{c}0.12 \\
(0.00)\end{array}$ & $\begin{array}{c}0.15 \\
(0.04)\end{array}$ & $\begin{array}{c}0.10 \\
(0.08)\end{array}$ & $\begin{array}{c}0.21 \\
(0.08)\end{array}$ & $\begin{array}{c}5.96 \\
(0.80)\end{array}$ & $\begin{array}{c}5.53 \\
(0.36)\end{array}$ & $\begin{array}{c}5.35 \\
(0.13)\end{array}$ & $\begin{array}{c}6.08 \\
(1.55)\end{array}$ & $\begin{array}{c}5.48 \\
(0.40)\end{array}$ & $\begin{array}{l}25.75 \\
(0.06)\end{array}$ \\
\hline $\mathrm{gO}$ & $\begin{array}{c}6.95 \\
(0.64)\end{array}$ & $\begin{array}{c}6.41 \\
(0.32)\end{array}$ & $\begin{array}{c}6.41 \\
(0.53)\end{array}$ & $\begin{array}{l}10.83 \\
(0.37)\end{array}$ & $\begin{array}{c}6.91 \\
(0.26)\end{array}$ & $\begin{array}{c}6.94 \\
(0.28)\end{array}$ & $\begin{array}{c}6.53 \\
(0.32)\end{array}$ & $\begin{array}{c}0.02 \\
(0.01)\end{array}$ & $\begin{array}{c}0.00 \\
(0.00)\end{array}$ & $\begin{array}{c}0.01 \\
(0.01)\end{array}$ & $\begin{array}{c}0.01 \\
(0.01)\end{array}$ & 0.00 & 0.01 & 02 & 0.00 & 0.01 & $\begin{array}{c}0.00 \\
(0.00)\end{array}$ & $\begin{array}{c}0.01 \\
(0.02)\end{array}$ & $\begin{array}{c}0.01 \\
(0.00)\end{array}$ & $\begin{array}{c}0.00 \\
(0.00)\end{array}$ & $\begin{array}{c}0.01 \\
(0.01)\end{array}$ & $\begin{array}{l}2.44 \\
(0.44)\end{array}$ & $\begin{array}{c}2.27 \\
(0.27)\end{array}$ & $\begin{array}{c}2.08 \\
(0.10)\end{array}$ & $\begin{array}{c}2.52 \\
(0.20)\end{array}$ & & $\begin{array}{l}15.30 \\
(0.35)\end{array}$ \\
\hline $\mathrm{AnO}$ & $\begin{array}{c}0.15 \\
(0.03)\end{array}$ & $\begin{array}{c}0.15 \\
(0.07)\end{array}$ & $\begin{array}{c}0.14 \\
(0.08)\end{array}$ & 0.18 & $\begin{array}{c}0.13 \\
(0.05)\end{array}$ & $\begin{array}{c}0.13 \\
(0.05)\end{array}$ & $\begin{array}{c}0.15 \\
(0.04)\end{array}$ & $\begin{array}{c}0.11 \\
(0.04)\end{array}$ & $\begin{array}{c}0.22 \\
(0.14)\end{array}$ & $\begin{array}{c}0.25 \\
(0.04)\end{array}$ & $\begin{array}{c}0.36 \\
(0.38)\end{array}$ & 0.50 & 0.19 & 0.19 & 0.10 & 0.08 & $\begin{array}{c}0.00 \\
(0.00)\end{array}$ & $\begin{array}{c}0.01 \\
(0.01)\end{array}$ & $\begin{array}{c}0.05 \\
(0.02)\end{array}$ & $\begin{array}{c}0.02 \\
(0.02)\end{array}$ & $\begin{array}{c}0.02 \\
(0.04)\end{array}$ & $\begin{array}{c}0.01 \\
(0.01)\end{array}$ & $\begin{array}{c}0.00 \\
(0.01)\end{array}$ & $\begin{array}{c}0.03 \\
(0.02)\end{array}$ & $\begin{array}{c}0.01 \\
(0.02)\end{array}$ & $\begin{array}{c}0.02 \\
(0.02)\end{array}$ & $\begin{array}{c}0.28 \\
(0.02)\end{array}$ \\
\hline $\mathrm{CaO}$ & $\begin{array}{c}0.54 \\
(0.01)\end{array}$ & $\begin{array}{c}0.68 \\
(0.38)\end{array}$ & $\begin{array}{c}0.42 \\
(0.32)\end{array}$ & $\begin{array}{c}9.02 \\
(0.96)\end{array}$ & $\begin{array}{c}0.58 \\
(0.29)\end{array}$ & $\begin{array}{c}0.58 \\
(0.20)\end{array}$ & $\begin{array}{c}0.74 \\
(0.22)\end{array}$ & $\begin{array}{l}23.04 \\
(0.14)\end{array}$ & $\begin{array}{l}23.59 \\
(0.42)\end{array}$ & $\begin{array}{l}23.12 \\
(0.03)\end{array}$ & $\begin{array}{l}22.84 \\
(0.42)\end{array}$ & 22.64 & 23.24 & 23.25 & 28.52 & 28.20 & $\begin{array}{c}0.06 \\
(0.03)\end{array}$ & $\begin{array}{c}0.06 \\
(0.03)\end{array}$ & $\begin{array}{c}0.08 \\
(0.04)\end{array}$ & $\begin{array}{c}0.07 \\
(0.02)\end{array}$ & $\begin{array}{c}0.04 \\
(0.01)\end{array}$ & $\begin{array}{c}0.03 \\
(0.01)\end{array}$ & $\begin{array}{c}0.02 \\
(0.03)\end{array}$ & $\begin{array}{c}0.01 \\
(0.01)\end{array}$ & $\begin{array}{c}0.06 \\
(0.05)\end{array}$ & $\begin{array}{c}0.02 \\
(0.02)\end{array}$ & $\begin{array}{c}0.06 \\
(0.04)\end{array}$ \\
\hline $\mathrm{Na}_{2} \mathrm{O}$ & $\begin{array}{c}7.19 \\
(0.14)\end{array}$ & $\begin{array}{c}7.23 \\
(0.16)\end{array}$ & $\begin{array}{c}7.35 \\
(0.13)\end{array}$ & $\begin{array}{c}2.58 \\
(0.65)\end{array}$ & $\begin{array}{c}7.17 \\
(0.15)\end{array}$ & $\begin{array}{c}7.12 \\
(0.12)\end{array}$ & $\begin{array}{c}7.11 \\
(0.09)\end{array}$ & $\begin{array}{c}0.00 \\
(0.00)\end{array}$ & $\begin{array}{c}0.05 \\
(0.02)\end{array}$ & $\begin{array}{c}0.01 \\
(0.00)\end{array}$ & $\begin{array}{c}0.01 \\
(0.01)\end{array}$ & 0.0 & 0.0 & 0.01 & 0.01 & 0.03 & $\begin{array}{l}11.97 \\
(0.04)\end{array}$ & $\begin{array}{l}12.13 \\
(0.33)\end{array}$ & $\begin{array}{l}11.60 \\
(0.54)\end{array}$ & $\begin{array}{l}12.19 \\
(0.16)\end{array}$ & & $\begin{array}{c}0.33 \\
(0.22)\end{array}$ & $\begin{array}{c}0.40 \\
(0.09)\end{array}$ & $\begin{array}{c}0.46 \\
(0.04)\end{array}$ & $\begin{array}{c}0.26 \\
(0.11)\end{array}$ & $\begin{array}{c}0.41 \\
(0.08)\end{array}$ & $\begin{array}{c}0.00 \\
(0.00)\end{array}$ \\
\hline o & $\begin{array}{c}0.01 \\
(0.01)\end{array}$ & $\begin{array}{c}0.02 \\
(0.01)\end{array}$ & $\begin{array}{c}0.02 \\
(0.00)\end{array}$ & $\begin{array}{c}0.31 \\
(0.07)\end{array}$ & $\begin{array}{c}0.02 \\
(0.01)\end{array}$ & $\begin{array}{c}0.01 \\
(0.01)\end{array}$ & $\begin{array}{c}0.03 \\
(0.02)\end{array}$ & $\begin{array}{c}0.01 \\
(0.01)\end{array}$ & $\begin{array}{c}0.01 \\
(0.01)\end{array}$ & $\begin{array}{c}0.01 \\
(0.00)\end{array}$ & $\begin{array}{c}0.00 \\
(0.00)\end{array}$ & 0.00 & 0.00 & 00 & 01 & 0.00 & $\begin{array}{c}0.02 \\
(0.01)\end{array}$ & $\begin{array}{c}0.02 \\
(0.00)\end{array}$ & $\begin{array}{c}0.03 \\
(0.02)\end{array}$ & $\begin{array}{c}0.02 \\
(0.01)\end{array}$ & & $\begin{array}{c}9.88 \\
(0.37)\end{array}$ & $\begin{array}{c}9.64 \\
(0.11)\end{array}$ & $\begin{array}{c}9.61 \\
(0.04)\end{array}$ & $\begin{array}{c}9.86 \\
(0.28)\end{array}$ & $\begin{array}{c}9.72 \\
(0.21)\end{array}$ & $\begin{array}{c}0.00 \\
(0.00)\end{array}$ \\
\hline Total & $\begin{array}{c}98.21 \\
(0.62)\end{array}$ & $\begin{array}{l}98.19 \\
(0.33)\end{array}$ & $\begin{array}{l}98.18 \\
(0.38)\end{array}$ & $\begin{array}{l}97.68 \\
(0.35)\end{array}$ & $\begin{array}{l}97.66 \\
(0.43)\end{array}$ & $\begin{array}{l}98.19 \\
(0.56)\end{array}$ & $\begin{array}{l}97.86 \\
(0.36)\end{array}$ & $\begin{array}{l}97.87 \\
(0.06)\end{array}$ & $\begin{array}{l}96.09 \\
(1.51)\end{array}$ & $\begin{array}{l}98.01 \\
(0.78)\end{array}$ & $\begin{array}{l}97.97 \\
(0.12)\end{array}$ & 97.57 & 98.11 & 98.68 & 98.60 & 98.33 & $\begin{array}{l}100.91 \\
(0.80)\end{array}$ & $\begin{array}{l}101.30 \\
(0.08)\end{array}$ & $\begin{array}{l}102.31 \\
(1.14)\end{array}$ & $\begin{array}{l}101.63 \\
(0.34)\end{array}$ & $\begin{array}{l}101.97 \\
(0.53)\end{array}$ & $\begin{array}{l}98.58 \\
(0.55)\end{array}$ & $\begin{array}{l}98.03 \\
(0.65)\end{array}$ & $\begin{array}{l}97.56 \\
(0.43)\end{array}$ & $\begin{array}{l}7.51 \\
.62)\end{array}$ & $\begin{array}{l}97.67 \\
(0.52)\end{array}$ & $\begin{array}{l}87.97 \\
(0.74)\end{array}$ \\
\hline & $\begin{array}{c}7.93 \\
(0.02)\end{array}$ & $\begin{array}{r}7.8 \\
(0.1\end{array}$ & $\begin{array}{c}7.98 \\
(0.04)\end{array}$ & $\begin{array}{c}7.47 \\
(0.05)\end{array}$ & $\begin{array}{c}7.91 \\
(0.11)\end{array}$ & $\begin{array}{c}7.9 \\
(0.0\end{array}$ & $\begin{array}{r}7.8 \\
(0.0\end{array}$ & $\begin{array}{c}2.99 \\
(0.00)\end{array}$ & $\begin{array}{c}2.99 \\
(0.05)\end{array}$ & $\begin{array}{c}2.97 \\
(0.01)\end{array}$ & $\begin{array}{c}2.98 \\
(0.02)\end{array}$ & 2.9 & 3.0 & 3.00 & 1.01 & 1.01 & $\begin{array}{c}2.8 \\
(0.01)\end{array}$ & $\begin{array}{c}2.97 \\
(0.02)\end{array}$ & $\begin{array}{c}3.00 \\
(0.05)\end{array}$ & $\begin{array}{c}2.97 \\
(0.02)\end{array}$ & $(0.8$ & $(0.04)$ & $\begin{array}{c}3.36 \\
(0.02)\end{array}$ & $\begin{array}{c}3.36 \\
(0.02)\end{array}$ & $\begin{array}{c}3.40 \\
(0.03)\end{array}$ & & $\begin{array}{c}2.75 \\
(0.04)\end{array}$ \\
\hline & $\begin{array}{c}0.01 \\
(0.00)\end{array}$ & $\begin{array}{c}0.01 \\
(0.01)\end{array}$ & $\begin{array}{c}0.01 \\
(0.00)\end{array}$ & $\begin{array}{c}0.01 \\
(0.01)\end{array}$ & $\begin{array}{c}0.02 \\
(0.01)\end{array}$ & $\begin{array}{c}0.01 \\
(0.01)\end{array}$ & $\begin{array}{c}0.02 \\
(0.01)\end{array}$ & $\begin{array}{c}0.01 \\
(0.00)\end{array}$ & $\begin{array}{c}0.01 \\
(0.01)\end{array}$ & $\begin{array}{c}0.01 \\
(0.00)\end{array}$ & $\begin{array}{c}0.02 \\
(0.02)\end{array}$ & 0.8 & $0 . c$ & 0.00 & 0.87 & 0. & $\begin{array}{c}0.00 \\
(0.00)\end{array}$ & $\begin{array}{c}0.00 \\
(0.00)\end{array}$ & $\begin{array}{c}0.00 \\
(0.00)\end{array}$ & $\begin{array}{c}0.00 \\
(0.00)\end{array}$ & & $\begin{array}{c}0.01 \\
(0.00)\end{array}$ & $\begin{array}{c}0.01 \\
(0.00)\end{array}$ & $\begin{array}{c}0.01 \\
(0.00)\end{array}$ & $\begin{array}{c}0.01 \\
(0.00)\end{array}$ & $\begin{array}{c}0.01 \\
(0.00)\end{array}$ & $\begin{array}{c}0.00 \\
(0.00)\end{array}$ \\
\hline 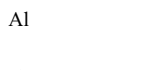 & $\begin{array}{c}1.37 \\
(0.05)\end{array}$ & $\begin{array}{c}1.44 \\
(0.08)\end{array}$ & $\begin{array}{c}1.37 \\
(0.08)\end{array}$ & $\begin{array}{c}0.80 \\
(0.11)\end{array}$ & $\begin{array}{c}1.34 \\
(0.12)\end{array}$ & $\begin{array}{c}1.36 \\
(0.09)\end{array}$ & $\begin{array}{c}1.34 \\
(0.07)\end{array}$ & $\begin{array}{c}2.26 \\
(0.03)\end{array}$ & $\begin{array}{c}2.07 \\
(0.04)\end{array}$ & $\begin{array}{c}2.19 \\
(0.04)\end{array}$ & $\begin{array}{c}2.24 \\
(0.03)\end{array}$ & 2. & 2.09 & & 0.10 & 0.10 & $\begin{array}{c}1.01 \\
(0.01)\end{array}$ & $\begin{array}{c}1.01 \\
(0.00)\end{array}$ & $\begin{array}{c}1.02 \\
(0.01)\end{array}$ & $\begin{array}{c}1.01 \\
(0.01)\end{array}$ & $\begin{array}{c}1.00 \\
(0.00)\end{array}$ & $\begin{array}{c}2.15 \\
(0.13)\end{array}$ & $\begin{array}{c}2.19 \\
(0.04)\end{array}$ & $\begin{array}{c}2.22 \\
(0.02)\end{array}$ & $\begin{array}{c}2.10 \\
(0.04)\end{array}$ & $\begin{array}{c}2.23 \\
(0.04)\end{array}$ & $\begin{array}{c}2.54 \\
(0.07)\end{array}$ \\
\hline $\mathrm{Cr}$ & $\begin{array}{c}0.00 \\
(0.00)\end{array}$ & $\begin{array}{c}0.00 \\
(0.00)\end{array}$ & $\begin{array}{c}0.00 \\
(0.00)\end{array}$ & $\begin{array}{c}0.00 \\
(0.00)\end{array}$ & $\begin{array}{c}0.00 \\
(0.00)\end{array}$ & $\begin{array}{c}0.00 \\
(0.00)\end{array}$ & $\begin{array}{c}0.00 \\
(0.00)\end{array}$ & $\begin{array}{c}0.00 \\
(0.00)\end{array}$ & $\begin{array}{c}0.00 \\
(0.00)\end{array}$ & $\begin{array}{c}0.00 \\
(0.00)\end{array}$ & $\begin{array}{c}0.00 \\
(0.00)\end{array}$ & 0.00 & 0.00 & 0.00 & 0.00 & 0.00 & $\begin{array}{c}0.00 \\
(0.00)\end{array}$ & $\begin{array}{c}0.00 \\
(0.00)\end{array}$ & $\begin{array}{c}0.00 \\
(0.00)\end{array}$ & $\begin{array}{c}0.00 \\
(0.00)\end{array}$ & $\begin{array}{c}0.00 \\
(0.00)\end{array}$ & $\begin{array}{c}0.00 \\
(0.00)\end{array}$ & $\begin{array}{c}0.00 \\
(0.00)\end{array}$ & $\begin{array}{c}0.00 \\
(0.00)\end{array}$ & $\begin{array}{c}0.00 \\
(0.00)\end{array}$ & $\begin{array}{c}0.00 \\
(0.00)\end{array}$ & $\begin{array}{c}0.00 \\
(0.00)\end{array}$ \\
\hline $\mathrm{Fe}^{*}$ & $\begin{array}{c}2.27 \\
(0.06)\end{array}$ & $\begin{array}{c}2.35 \\
(0.10)\end{array}$ & $\begin{array}{c}2.29 \\
(0.04)\end{array}$ & $\begin{array}{c}2.40 \\
(0.04)\end{array}$ & $\begin{array}{c}2.32 \\
(0.04)\end{array}$ & $\begin{array}{c}2.30 \\
(0.07)\end{array}$ & $\begin{array}{c}2.43 \\
(0.12)\end{array}$ & $\begin{array}{c}0.79 \\
(0.01)\end{array}$ & $\begin{array}{c}0.87 \\
(0.02)\end{array}$ & $\begin{array}{c}0.85 \\
(0.03)\end{array}$ & $\begin{array}{c}0.81 \\
(0.03)\end{array}$ & 0.8 & 0.90 & 0.90 & 0.02 & 0.02 & $\begin{array}{c}0.00 \\
(0.00)\end{array}$ & $\begin{array}{c}0.00 \\
(0.00)\end{array}$ & $\begin{array}{c}0.01 \\
(0.00)\end{array}$ & $\begin{array}{c}0.00 \\
(0.00)\end{array}$ & $\begin{array}{c}0.01 \\
(0.00)\end{array}$ & $\begin{array}{c}0.33 \\
(0.05)\end{array}$ & $\begin{array}{c}0.30 \\
(0.02)\end{array}$ & $\begin{array}{c}0.29 \\
(0.01)\end{array}$ & $\begin{array}{c}0.34 \\
(0.09)\end{array}$ & $\begin{array}{c}0.30 \\
(0.02)\end{array}$ & $\begin{array}{c}2.27 \\
(0.02)\end{array}$ \\
\hline Gg & $\begin{array}{c}1.47 \\
(0.14)\end{array}$ & $\begin{array}{c}1.36 \\
(0.06)\end{array}$ & $\begin{array}{c}1.35 \\
(0.12)\end{array}$ & $\begin{array}{c}2.38 \\
(0.09)\end{array}$ & $\begin{array}{c}1.47 \\
(0.06)\end{array}$ & $\begin{array}{c}1.47 \\
(0.07)\end{array}$ & $\begin{array}{c}1.40 \\
(0.07)\end{array}$ & $\begin{array}{c}0.00 \\
(0.00)\end{array}$ & $\begin{array}{c}0.00 \\
(0.00)\end{array}$ & $\begin{array}{c}0.00 \\
(0.00)\end{array}$ & $\begin{array}{c}0.00 \\
(0.00)\end{array}$ & 0. & 0. & 0.00 & 0.00 & 0.0 & $\begin{array}{c}0.00 \\
(0.00)\end{array}$ & $\begin{array}{c}0.00 \\
(0.00)\end{array}$ & $\begin{array}{c}0.00 \\
(0.00)\end{array}$ & $\begin{array}{c}0.00 \\
(0.00)\end{array}$ & $\begin{array}{c}0.00 \\
(0.00)\end{array}$ & $\begin{array}{c}0.24 \\
(0.05)\end{array}$ & $\begin{array}{c}0.22 \\
(0.03)\end{array}$ & $\begin{array}{c}0.20 \\
(0.01)\end{array}$ & $\begin{array}{c}0.25 \\
(0.02)\end{array}$ & $\begin{array}{c}0.21 \\
(0.02)\end{array}$ & $\begin{array}{c}2.40 \\
(0.03)\end{array}$ \\
\hline $\mathrm{Mn}$ & $\begin{array}{c}0.02 \\
(0.00)\end{array}$ & $\begin{array}{c}0.02 \\
(0.01)\end{array}$ & $\begin{array}{c}0.02 \\
(0.01)\end{array}$ & $\begin{array}{c}0.02 \\
(0.01)\end{array}$ & $\begin{array}{c}0.02 \\
(0.01)\end{array}$ & $\begin{array}{c}0.02 \\
(0.01)\end{array}$ & $\begin{array}{c}0.02 \\
(0.00)\end{array}$ & $\begin{array}{c}0.01 \\
(0.00)\end{array}$ & $\begin{array}{c}0.02 \\
(0.01)\end{array}$ & $\begin{array}{c}0.02 \\
(0.00)\end{array}$ & $\begin{array}{c}0.02 \\
(0.03)\end{array}$ & 0.03 & 0.01 & 0.01 & 0.00 & 0 & $\begin{array}{c}0.00 \\
(0.00)\end{array}$ & $\begin{array}{c}0.00 \\
(0.00)\end{array}$ & $\begin{array}{c}0.00 \\
(0.00)\end{array}$ & $\begin{array}{c}0.00 \\
(0.00)\end{array}$ & $\begin{array}{c}0.00 \\
(0.00)\end{array}$ & $\begin{array}{c}0.00 \\
(0.00)\end{array}$ & $\begin{array}{c}0.00 \\
(0.00)\end{array}$ & $\begin{array}{c}0.00 \\
(0.00)\end{array}$ & $\begin{array}{c}0.00 \\
(0.00)\end{array}$ & $\begin{array}{c}0.00 \\
(0.00)\end{array}$ & $\begin{array}{c}0.02 \\
(0.01)\end{array}$ \\
\hline $\mathrm{Ca}$ & $\begin{array}{c}0.08 \\
(0.00)\end{array}$ & $\begin{array}{c}0.10 \\
(0.06)\end{array}$ & $\begin{array}{c}0.06 \\
(0.05)\end{array}$ & $\begin{array}{c}1.43 \\
(0.16)\end{array}$ & $\begin{array}{c}0.09 \\
(0.04)\end{array}$ & $\begin{array}{c}0.09 \\
(0.03)\end{array}$ & $\begin{array}{c}0.11 \\
(0.04)\end{array}$ & $\begin{array}{c}1.94 \\
(0.01)\end{array}$ & $\begin{array}{c}2.03 \\
(0.00)\end{array}$ & $\begin{array}{c}1.95 \\
(0.02)\end{array}$ & $\begin{array}{c}1.92 \\
(0.03)\end{array}$ & 1.92 & 1.96 & 1.95 & 1.00 & 0.99 & $\begin{array}{c}0.00 \\
(0.00)\end{array}$ & $\begin{array}{c}0.00 \\
(0.00)\end{array}$ & $\begin{array}{c}0.00 \\
(0.00)\end{array}$ & $\begin{array}{c}0.00 \\
(0.00)\end{array}$ & $\begin{array}{c}0.00 \\
(0.00)\end{array}$ & $\begin{array}{c}0.00 \\
(0.00)\end{array}$ & $\begin{array}{c}0.00 \\
(0.00)\end{array}$ & $\begin{array}{c}0.00 \\
(0.00)\end{array}$ & $\begin{array}{c}0.00 \\
(0.00)\end{array}$ & $\begin{array}{c}0.00 \\
(0.00)\end{array}$ & $\begin{array}{c}0.01 \\
(0.01)\end{array}$ \\
\hline $\mathrm{Na}$ & $\begin{array}{c}1.98 \\
(0.03)\end{array}$ & $\begin{array}{c}2.00 \\
(0.03)\end{array}$ & $\begin{array}{c}2.02 \\
(0.03)\end{array}$ & $\begin{array}{c}0.74 \\
(0.18)\end{array}$ & $\begin{array}{c}1.99 \\
(0.03)\end{array}$ & $\begin{array}{c}1.96 \\
(0.03)\end{array}$ & $\begin{array}{c}1.98 \\
(0.02)\end{array}$ & $\begin{array}{c}0.00 \\
(0.00)\end{array}$ & $\begin{array}{c}0.01 \\
(0.00)\end{array}$ & $\begin{array}{c}0.00 \\
(0.00)\end{array}$ & $\begin{array}{c}0.00 \\
(0.00)\end{array}$ & 0.0 & 0.00 & 0.00 & 0.0 & 0.00 & $\begin{array}{c}1.00 \\
(0.01)\end{array}$ & $\begin{array}{c}1.01 \\
(0.02)\end{array}$ & $\begin{array}{c}0.96 \\
(0.05)\end{array}$ & $\begin{array}{c}1.01 \\
(0.01)\end{array}$ & $\begin{array}{c}1.02 \\
(0.01)\end{array}$ & $\begin{array}{c}0.04 \\
(0.03)\end{array}$ & $\begin{array}{c}0.05 \\
(0.01)\end{array}$ & $\begin{array}{c}0.06 \\
(0.00)\end{array}$ & $\begin{array}{c}0.03 \\
(0.01)\end{array}$ & $\begin{array}{c}0.05 \\
(0.01)\end{array}$ & $\begin{array}{c}0.00 \\
(0.00)\end{array}$ \\
\hline & $\begin{array}{c}0.00 \\
(0.00)\end{array}$ & $\begin{array}{c}0.00 \\
(0.00)\end{array}$ & $\begin{array}{c}0.00 \\
(0.00)\end{array}$ & $\begin{array}{c}0.06 \\
(0.01)\end{array}$ & $\begin{array}{c}0.00 \\
(0.00)\end{array}$ & $\begin{array}{c}0.00 \\
(0.00)\end{array}$ & $\begin{array}{c}0.01 \\
(0.00)\end{array}$ & $\begin{array}{c}0.00 \\
(0.00)\end{array}$ & $\begin{array}{c}0.00 \\
(0.00)\end{array}$ & $\begin{array}{c}0.00 \\
(0.00)\end{array}$ & $\begin{array}{c}0.00 \\
(0.00)\end{array}$ & 0. & $0 . c$ & & 0.00 & 0. & $\begin{array}{c}0.00 \\
(0.00)\end{array}$ & & $\begin{array}{c}0.00 \\
(0.00)\end{array}$ & $\begin{array}{c}0.00 \\
(0.00)\end{array}$ & & & $\begin{array}{c}0.80 \\
(0.01)\end{array}$ & $\begin{array}{c}0.80 \\
(0.00)\end{array}$ & $\begin{array}{c}0.83 \\
(0.03)\end{array}$ & $\begin{array}{c}0.82 \\
(0.02)\end{array}$ & $\begin{array}{c}0.00 \\
(0.00)\end{array}$ \\
\hline Total & $\begin{array}{l}15.13 \\
(0.02)\end{array}$ & $\begin{array}{l}15.16 \\
(0.17)\end{array}$ & $\begin{array}{l}15.11 \\
(0.07)\end{array}$ & $\begin{array}{l}15.29 \\
(0.03)\end{array}$ & $\begin{array}{l}15.16 \\
(0.04)\end{array}$ & $\begin{array}{l}15.14 \\
(0.06)\end{array}$ & $\begin{array}{l}15.18 \\
(0.08)\end{array}$ & $\begin{array}{c}8.00 \\
(0.00)\end{array}$ & $\begin{array}{c}8.00 \\
(0.00)\end{array}$ & $\begin{array}{c}8.00 \\
(0.00)\end{array}$ & $\begin{array}{c}8.00 \\
(0.00)\end{array}$ & 8.00 & 8.00 & 8.00 & 3.00 & 3.00 & $\begin{array}{c}5.00 \\
(0.00)\end{array}$ & $\begin{array}{c}5.00 \\
(0.00)\end{array}$ & $\begin{array}{c}5.00 \\
(0.00)\end{array}$ & $\begin{array}{c}5.00 \\
(0.00)\end{array}$ & $\begin{array}{c}5.00 \\
(0.00)\end{array}$ & $\begin{array}{c}6.97 \\
(0.03)\end{array}$ & $\begin{array}{c}6.95 \\
(0.01)\end{array}$ & $\begin{array}{c}6.95 \\
(0.01)\end{array}$ & $\begin{array}{c}6.97 \\
(0.05)\end{array}$ & $\begin{array}{c}6.96 \\
(0.02)\end{array}$ & $\begin{array}{l}10.86 \\
(0.01)\end{array}$ \\
\hline
\end{tabular}


Table 4. Average composition of major elements (ox. wt \%) and of recalculated structural formulae of minerals composing mafic rocks from the Ophiolitic Complex (numbers in brackets are standard deviations)

\begin{tabular}{|c|c|c|c|c|c|c|c|c|c|c|c|c|}
\hline \multirow{3}{*}{$\begin{array}{l}\text { Sample Mineral } \\
\text { Microstructural } \\
\text { position } \\
\text { No. analyses }\end{array}$} & \multicolumn{6}{|c|}{ Amphibole } & \multicolumn{2}{|c|}{ Titanite } & \multicolumn{2}{|c|}{ Plagioclase } & \multicolumn{2}{|c|}{ Phengite } \\
\hline & \multicolumn{3}{|c|}{ AK33A } & \multicolumn{3}{|c|}{ AK36 } & AK33B & AK36 & AK33A & AK36 & AK33A & \multirow[t]{2}{*}{ AK36 } \\
\hline & $\begin{array}{c}\mathrm{Amp}_{2} \text { fine } \\
3\end{array}$ & $\begin{array}{c}\mathrm{Amp}_{2} \text { Core } \\
3\end{array}$ & $\underset{2}{\mathrm{Amp}_{2} \operatorname{Rim}}$ & $\begin{array}{c}\mathrm{Amp}_{2} \text { fine } \\
3\end{array}$ & $\begin{array}{c}\mathrm{Amp}_{2} \text { Core } \\
3\end{array}$ & $\begin{array}{c}\mathrm{Amp}_{2} \operatorname{Rim} \\
6\end{array}$ & $\begin{array}{c}\mathrm{Ttn}_{2} \\
3\end{array}$ & $\begin{array}{c}\mathrm{Ttn}_{2} \\
5\end{array}$ & $\begin{array}{c}\text { Ab Porph } \\
5\end{array}$ & $\begin{array}{c}\text { Ab Porph } \\
12\end{array}$ & $\begin{array}{c}\mathrm{Wm}_{2} \\
5\end{array}$ & \\
\hline $\mathrm{SiO}_{2}$ & $55.20(0.24)$ & $55.07(0.20)$ & $55.47(0.18)$ & $55.60(0.35)$ & $55.74(0.32)$ & $55.74(0.46)$ & $30.78(0.31)$ & $30.73(0.24)$ & $68.82(0.28)$ & $68.87(0.30)$ & $53.56(0.60)$ & $53.68(0.87)$ \\
\hline $\mathrm{TiO}_{2}$ & $0.07(0.01)$ & $0.04(0.04)$ & $0.03(0.03)$ & $0.14(0.16)$ & $0.06(0.01)$ & $0.03(0.04)$ & $36.39(1.38)$ & $36.68(1.24)$ & $0.01(0.02)$ & $0.01(0.01)$ & $0.08(0.05)$ & $0.11(0.02)$ \\
\hline $\mathrm{A}_{12} \mathrm{O}_{3}$ & $2.36(0.79)$ & $2.29(0.08)$ & $2.31(0.30)$ & $2.38(0.66)$ & $2.59(0.42)$ & $2.10(0.46)$ & $1.74(0.63)$ & $1.44(0.60)$ & $19.56(0.14)$ & $19.64(0.20)$ & $24.93(0.85)$ & $24.57(0.88)$ \\
\hline $\mathrm{Cr}_{2} \mathrm{O}_{3}$ & $0.06(0.04)$ & $0.06(0.05)$ & $0.05(0.03)$ & $0.03(0.04)$ & $0.07(0.04)$ & $0.05(0.04)$ & $0.01(0.01)$ & $0.04(0.05)$ & $0.00(0.00)$ & $0.01(0.01)$ & $0.11(0.06)$ & $0.15(0.03)$ \\
\hline $\mathrm{FeO}^{*}$ & $19.10(0.43)$ & $20.73(1.17)$ & $20.64(0.55)$ & $19.20(0.12)$ & $19.75(0.43)$ & $19.21(1.56)$ & $1.09(0.34)$ & $1.13(0.14)$ & $0.11(0.05)$ & $0.06(0.04)$ & $5.14(0.70)$ & $4.78(1.33)$ \\
\hline $\mathrm{MgO}$ & $10.92(0.66)$ & $9.79(0.87)$ & $9.73(0.03)$ & $10.43(0.59)$ & $10.01(0.27)$ & $10.67(0.74)$ & $0.04(0.03)$ & $0.04(0.02)$ & $0.01(0.01)$ & $0.01(0.01)$ & $3.50(0.07)$ & $3.73(0.07)$ \\
\hline $\mathrm{MnO}$ & $0.15(0.00)$ & $0.15(0.03)$ & $0.12(0.01)$ & $0.15(0.02)$ & $0.12(0.01)$ & $0.15(0.04)$ & $0.02(0.02)$ & $0.01(0.01)$ & $0.01(0.02)$ & $0.02(0.02)$ & $0.01(0.02)$ & $0.00(0.00)$ \\
\hline $\mathrm{CaO}$ & $3.47(1.58)$ & $2.26(0.68)$ & $1.64(0.18)$ & $2.01(0.78)$ & $1.12(0.20)$ & $2.31(1.83)$ & $28.06(0.21)$ & $28.07(0.28)$ & $0.01(0.01)$ & $0.01(0.01)$ & $0.04(0.03)$ & $0.01(0.01)$ \\
\hline $\mathrm{Na}_{2} \mathrm{O}$ & $5.36(0.77)$ & $6.23(0.33)$ & $6.42(0.02)$ & $6.44(0.54)$ & $6.68(0.05)$ & $6.06(0.95)$ & $0.02(0.02)$ & $0.05(0.06)$ & $11.54(0.10)$ & $11.57(0.09)$ & $0.11(0.04)$ & $0.11(0.05)$ \\
\hline $\mathrm{K}_{2} \mathrm{O}$ & $0.08(0.05)$ & $0.05(0.02)$ & $0.04(0.01)$ & $0.03(0.00)$ & $0.02(0.01)$ & $0.03(0.01)$ & $0.09(0.06)$ & $0.07(0.10)$ & $0.03(0.01)$ & $0.00(0.01)$ & $9.79(0.53)$ & $9.79(0.17)$ \\
\hline Total & $96.78(0.20)$ & $96.67(0.53)$ & $96.43(0.32)$ & $96.42(0.20)$ & $96.18(0.38)$ & $96.35(0.32)$ & $98.25(0.28)$ & $98.25(0.42)$ & $100.10(0.46)$ & $100.19(0.47)$ & $97.26(0.58)$ & $96.93(0.51)$ \\
\hline $\mathrm{Si}$ & $7.89(0.02)$ & $7.91(0.05)$ & $7.97(0.01)$ & $7.96(0.06)$ & $7.99(0.03)$ & $7.98(0.04)$ & $1.01(0.01)$ & $1.01(0.01)$ & $3.01(0.01)$ & $3.01(0.01)$ & $3.52(0.02)$ & $3.53(0.02)$ \\
\hline $\mathrm{Ti}$ & $0.01(0.00)$ & $0.00(0.00)$ & $0.00(0.00)$ & $0.01(0.02)$ & $0.01(0.00)$ & $0.00(0.00)$ & $0.90(0.03)$ & $0.91(0.03)$ & $0.00(0.00)$ & $0.00(0.00)$ & $0.00(0.00)$ & $0.01(0.00)$ \\
\hline $\mathrm{Al}$ & $0.40(0.13)$ & $0.39(0.01)$ & $0.39(0.05)$ & $0.40(0.11)$ & $0.44(0.07)$ & $0.35(0.08)$ & $0.07(0.02)$ & $0.06(0.02)$ & $1.01(0.00)$ & $1.01(0.01)$ & $1.93(0.05)$ & $1.91(0.05)$ \\
\hline $\mathrm{Cr}$ & $0.01(0.00)$ & $0.01(0.01)$ & $0.01(0.00)$ & $0.00(0.00)$ & $0.01(0.00)$ & $0.01(0.00)$ & $0.00(0.00)$ & $0.00(0.00)$ & $0.00(0.00)$ & $0.00(0.00)$ & $0.01(0.00)$ & $0.01(0.00)$ \\
\hline $\mathrm{Fe}^{*}$ & $2.28(0.05)$ & $2.49(0.15)$ & $2.48(0.06)$ & $2.30(0.02)$ & $2.37(0.06)$ & $2.30(0.19)$ & $0.03(0.01)$ & $0.03(0.00)$ & $0.00(0.00)$ & $0.00(0.00)$ & $0.28(0.04)$ & $0.26(0.08)$ \\
\hline $\mathrm{Mg}$ & $2.33(0.14)$ & $2.10(0.17)$ & $2.08(0.00)$ & $2.23(0.13)$ & $2.14(0.05)$ & $2.28(0.15)$ & $0.00(0.00)$ & $0.00(0.00)$ & $0.00(0.00)$ & $0.00(0.00)$ & $0.34(0.01)$ & $0.37(0.01)$ \\
\hline $\mathrm{Mn}$ & $0.02(0.00)$ & $0.02(0.00)$ & $0.02(0.00)$ & $0.02(0.00)$ & $0.01(0.00)$ & $0.02(0.00)$ & $0.00(0.00)$ & $0.00(0.00)$ & $0.00(0.00)$ & $0.00(0.00)$ & $0.00(0.00)$ & $0.00(0.00)$ \\
\hline $\mathrm{Ca}$ & $0.53(0.24)$ & $0.35(0.10)$ & $0.25(0.03)$ & $0.31(0.12)$ & $0.17(0.03)$ & $0.35(0.28)$ & $0.99(0.01)$ & $0.99(0.01)$ & $0.00(0.00)$ & $0.00(0.00)$ & $0.00(0.00)$ & $0.00(0.00)$ \\
\hline $\mathrm{Na}$ & $1.49(0.21)$ & $1.74(0.10)$ & $1.79(0.01)$ & $1.79(0.15)$ & $1.86(0.01)$ & $1.68(0.27)$ & $0.00(0.00)$ & $0.00(0.00)$ & $0.98(0.01)$ & $0.98(0.01)$ & $0.01(0.01)$ & $0.01(0.01)$ \\
\hline $\mathrm{K}$ & $0.01(0.01)$ & $0.01(0.00)$ & $0.01(0.00)$ & $0.01(0.00)$ & $0.00(0.00)$ & $0.01(0.00)$ & $0.00(0.00)$ & $0.00(0.00)$ & $0.00(0.00)$ & $0.00(0.00)$ & $0.82(0.05)$ & $0.82(0.01)$ \\
\hline Total & $14.96(0.02)$ & $15.01(0.03)$ & $14.99(0.01)$ & $15.03(0.03)$ & $15.00(0.01)$ & $14.99(0.06)$ & $3.00(0.00)$ & $3.00(0.00)$ & $5.00(0.00)$ & $5.00(0.00)$ & $6.92(0.05)$ & $6.92(0.03)$ \\
\hline
\end{tabular}


respect to white micas of mafic rocks from the CGC and MC. As already discussed by Zanchi et al. (2015), this comparison suggests that phengitic micas of metapillow basalts likely re-equilibrated with riebeckite under blueschist-facies conditions during a prograde, relatively HP, metamorphism.

\section{5.b.4. Thermodynamic modelling}

A $P-T$ isochemical section was calculated with PERPLE_X software (Connolly, 2005), considering the bulk composition of sample AK14b and $\mathrm{H}_{2} \mathrm{O}$ saturated conditions, using the thermodynamic database and equation of state for $\mathrm{H}_{2} \mathrm{O}$ of Holland \& Powell (1998, upgraded 2002). No phase has been excluded from the calculation. Solution models of Holland \& Powell $(1998,2003)$ were used for olivine, orthopyroxene, clinopyroxene, garnet, phengite, feldspar, epidote, chlorite and biotite. Two different solution models have been used to model $\mathrm{Ca}$-amphibole and Na-amphibole from Dale et al. (2005), which require $\mathrm{Fe}_{2} \mathrm{O}_{3}$ and $\mathrm{FeO}$ concentrations. $\mathrm{Fe}^{+3}$-rich minerals such as epidote also occurs in both HP-LT and syn$\mathrm{S}_{2}$ metamorphic event, and the use of a solid solution model for epidote is crucial to model their possible stability fields. For this reason, we measured the $\mathrm{FeO}$ and $\mathrm{Fe}_{2} \mathrm{O}_{3}$ concentration of the whole rock by chemical titration and considered $\mathrm{FeO}$ and $\mathrm{Fe}_{2} \mathrm{O}_{3}$ as distinct chemical components in the thermodynamic forward modelling. The phase diagram section reported in Figure 7 shows that the (pre- $\left.\mathrm{S}_{2}\right) \mathrm{Cpx}$-free mineral association $\mathrm{Na}-\mathrm{Amp}_{1}+\mathrm{Wm}_{1}+\mathrm{Ttn}_{1}+\mathrm{Ep}_{1}+\mathrm{Pl}_{1}$ is stable in a narrow $P-T$ range between 0.8 and $0.95 \mathrm{GPa}$ and 410 $460^{\circ} \mathrm{C}$, respectively. The appearance of chlorite in the $\mathrm{S}_{2} \mathrm{Cpx}$-free and Bio-free mineral assemblage (blue text in Fig. 7) $\mathrm{Amp}_{2}$ (core) $+\mathrm{Chl}+\mathrm{Wm}_{2}+\mathrm{Ep}_{2}$ (core) $+\mathrm{Pl}_{2}$ occurs at similar temperature conditions and lower pressures, down to $0.65 \mathrm{GPa}$.

Comparing the Morghab metamorphic peak conditions with those of the Chah Gorbeh and Ophiolite units $\left(0.75-0.85 \mathrm{GPa}\right.$ and $\left.350-400^{\circ} \mathrm{C}\right)$ reported by Zanchi et al. (2015), the MC mafic schists experienced HP metamorphism before the development of the $\mathrm{S}_{2}$ foliation as will be discussed later. It is worth noting that the thermodynamic modelling results are strongly supported by the microstructural evidence (Fig. 5).

Thermodynamic modelling of the stability of solid solutions with $\mathrm{Fe}^{3+}$-bearing end-member minerals (i.e. epidote and Na-amphibole) requires the assumption that the bulk $\mathrm{Fe}^{2+} / \mathrm{Fe}^{3+}$ ratio was constant during the entire metamorphic cycle of the rock. We therefore compared the $P-T$ isochemical section of Figure 7 with a $T-f \mathrm{O}_{2}$ isochemical section calculated at $0.9 \mathrm{GPa}$, which is portrayed in Figure 8. The starting composition is the same as that used in Figure 7, but considering $\mathrm{FeO}$ as total iron. The peak (pre- $\mathrm{S}_{2}$ ) Na$\mathrm{Amp}_{1}+\mathrm{Wm}_{1}+\mathrm{Ttn}_{1}+\mathrm{Ep}_{1}+\mathrm{Pl}_{1}$ mineral association is stable at $460^{\circ} \mathrm{C}$ and $\log f \mathrm{O}_{2} \cong-25.5$, corresponding to FMQ-1 (FMQ is the reference fayalite-magnetitequartz buffer). It is worth noting that the stability of epidote is strongly influenced by the oxidation state of the bulk composition, therefore by the $\mathrm{Fe}^{2+} / \mathrm{Fe}^{3+}$ ratio of the rock. The $f \mathrm{O}_{2}$ value calculated from forward modelling enables a back calculation of the expected $\mathrm{FeO}$ and $\mathrm{Fe}_{2} \mathrm{O}_{3}$ content of the whole rock using the equation of Kress \& Carmichael (1988). The measured $\mathrm{Fe}^{2+} / \mathrm{Fe}^{3+}$ ratio at $T=450{ }^{\circ} \mathrm{C}$ and $P=1$ atm would be stable at $\log f \mathrm{O}_{2}=-24$, which is perfectly consistent with the values calculated in Figure 8, if we consider that $\mathrm{fO}_{2}$ slightly decreases with increasing pressure. We can therefore assume that the $\mathrm{Fe}^{2+} / \mathrm{Fe}^{3+}$ content used to reconstruct the $P-T$ stability field of epidoteand Na-amphibole-bearing assemblage is representative of the composition of the rock at the pressure-peak conditions.

\section{Discussion}

The three main units constituting the gross part of the AMC show a complex structural pattern that is the result of at least three deformation events. The MC, CGC and $\mathrm{OC}$ were refolded together during the last deformation stage $\left(\mathrm{D}_{3}\right)$ and overthrust by the Lakh Marble unit (Zanchi et al. 2015). $\mathrm{D}_{3}$ structure developments occurred at non- or very low-grade metamorphic conditions (Fig. 3e). The metamorphic evolution of the three units should then be related to pre- $\mathrm{D}_{3}$ deformation stages.

Field structural analyses (Zanchi et al. 2015) individuated a regional foliation $\left(S_{2}\right)$ that is almost parallel in the MC and CGC units, suggesting that it is probably related to the same deformation event that affected the two units during or after their tectonic coupling. However, the detailed study of microstructures and mineral chemistry of blueschist-facies mineral assemblages in the AMC rocks presented in this study suggests that the three units experienced tectonometamorphic evolutions that differed in some stages.

The spatial relationships between compositionally distinct amphiboles in the MC, CGC and OC mafic rocks point to a pre- $\mathrm{S}_{2}$ (regional foliation) pressure peak in the MC. Amphiboles $\left(\mathrm{Amp}_{1}\right)$ included in plagioclase $\left(\mathrm{Pl}_{1}\right)$ and epidote $\left(\mathrm{Ep}_{2}\right)$ porphyroblasts are glaucophane or ferroglaucophane (Table 2; Fig. 6), whereas $\mathrm{Amp}_{2}$, aligned along the main foliation $\left(\mathrm{S}_{2}\right)$, are calcic amphiboles $\left(\mathrm{Amp}_{2}\right.$ in Table 2). Deformation-recrystallization relationships thus indicate that peak pressure $(0.80-0.95 \mathrm{GPa}$ at $T$ of 410 $450^{\circ} \mathrm{C}$, Fig. 7) was reached by the MC mafic schists before the development of the $\mathrm{S}_{2}$ foliation. Calcic amphiboles $\left(\mathrm{Amp}_{2}\right)$ and epidotes $\left(\mathrm{Ep}_{2}\right)$ syn-kinematic with the $\mathrm{S}_{2}$ foliation display in turn a core-rim compositional zoning indicative of a pressure increase during or after the $\mathrm{D}_{2}$ deformation event. Based on these data we can define a clockwise $P-T$ path for the AMC (Fig. 9), with a blueschist-facies stage followed by greenschist re-equilibration at lower pressure and later underplating to greater depths. The amphibole chemical composition suggests that the late- $\mathrm{S}_{2}$ metamorphic 


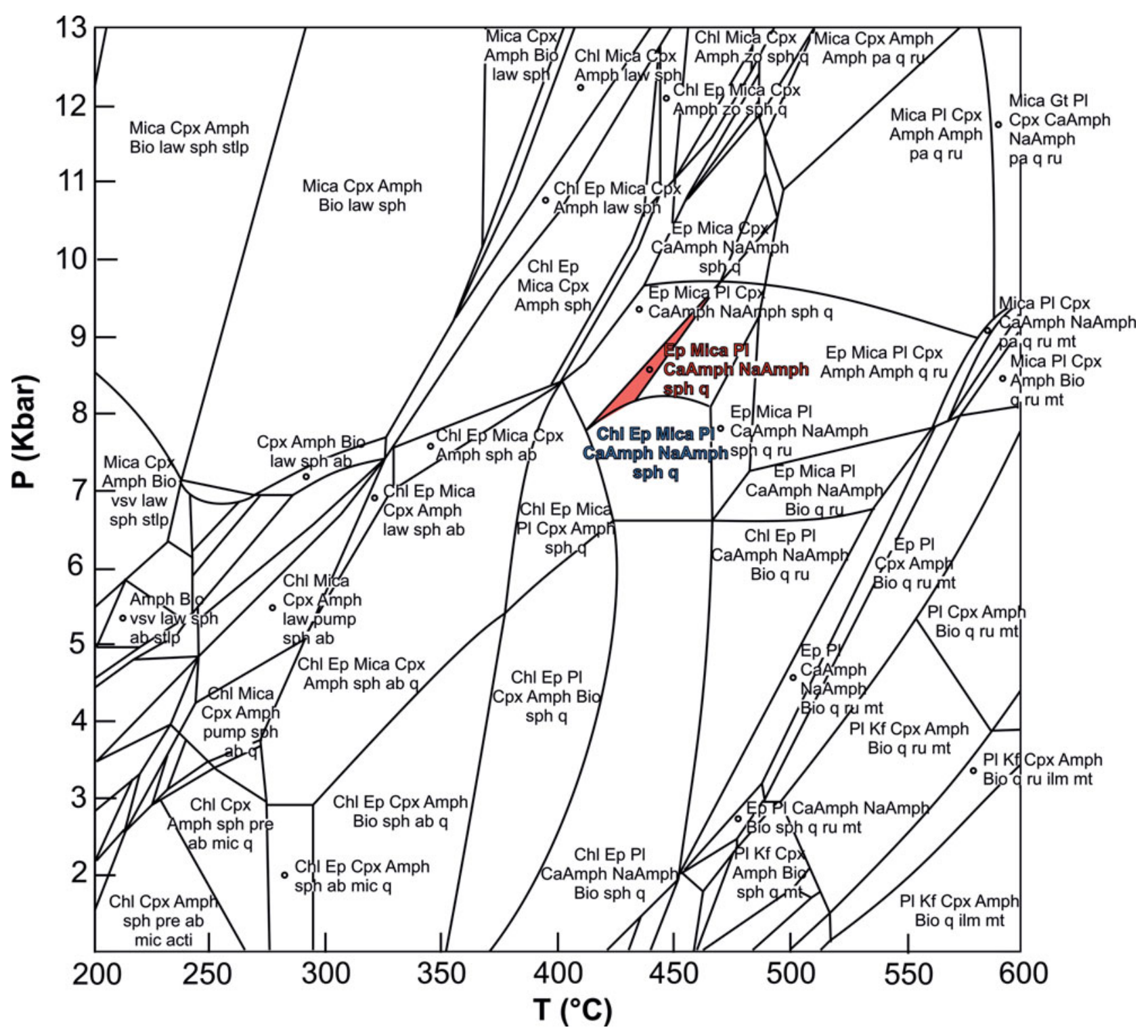

Figure 7. (Colour online) Isochemical $P-T$ section for Morghab meta-basalt calculated in the system $\mathrm{SiO}_{2}(50.27)-\mathrm{Al}_{2} \mathrm{O}_{3}(14.42)-$ $\mathrm{MgO}(5.61)-\mathrm{CaO}(7.27)-\mathrm{Na}_{2} \mathrm{O}(4.53)-\mathrm{K}_{2} \mathrm{O}(0.82)-\mathrm{TiO}_{2}(1.41)-\mathrm{FeO}(8.57)-\mathrm{Fe}_{2} \mathrm{O}_{3}$ (3.43). Values in brackets are oxide wt. \% The red area shows the hypothetical stability field of the relict HP paragenesis preserved in porphyroblastic epidote, as evidenced by microstructural and mineral chemistry analyses. Abbreviations for solid solution models: Amph - Ca-amphiboles and Na-amphiboles; Bio - biotite; Chl - chlorite; Ep - epidote; Cpx - clinopyroxene; Gt - garnet; Kfs - alkali feldspar; Mica - phengite; Pl - ternary feldspar. Abbreviations for phases: ab - albite; acti - actinolite; fctd - Fe-chloritoid; ilm - ilmenite; law - lawsonite; mic - microcline; $\mathrm{mt}$ - magnetite; q-quartz; pa - paragonite; pre - prehnite; pump - pumpellyite; ru - rutile; sph - titanite (sphene); stlp - stilpnomelane; vsv - vesuvianite; zo - zoisite. The paragenesis at pressure peak observed in thin-section is highlighted in red. The same equilibrium phase assemblage with chlorite in addition (blue text in figure) occurs at the same $T$, but a lower pressure with respect to the chloritefree one.

stage did not reach the pressure-peak experienced by the $\mathrm{MC}$ mafic rocks during the pre- $\mathrm{D}_{2}$ evolution.

The CGC and OC mafic rocks display a contrasting metamorphic evolution. HP-LT metamorphism is demonstrated to have followed a greenschist-facies metamorphic event as shown by microstructures and mineral chemistry. Sodic amphibole $\left(\mathrm{Amp}_{2}\right)$ in CGC rocks is aligned along the main foliation $\left(\mathrm{S}_{2}\right.$, Fig. 5), whereas low- $P$ actinolitic amphibole $\left(\mathrm{Amp}_{1}\right)$ is preserved as inclusions within $\mathrm{Pl}_{1}$ and $\mathrm{Ep}_{1}$ porphyroblasts which are pre-kinematic with respect to $\mathrm{S}_{2}$. The compositional variation of epidote, with syn- $\mathrm{S}_{2} \mathrm{Ep}_{2}$ enriched in $\mathrm{Fe}^{3+}$ with respect to $\mathrm{Ep}_{1}$, and the growth of rutile instead of titanite as the main Ti-bearing phase, could be considered as clues that point to a $P$ increase during the $\mathrm{D}_{2}$ deformation event. Nevertheless, special care should be taken as at low to medium pressure, the compositional variation of epidote also depends on $f \mathrm{O}_{2}$, coexisting $\mathrm{Ca}-\mathrm{Al}$ silicates and $\mathrm{H}_{2} \mathrm{O}$-content (e.g. Grapes \& Hoskin, 2004).

The meta-pillow basalts provide less information on the deformation-metamorphism relationships as they are substantially non-deformed. A poorly developed primary foliation $\left(\mathrm{S}_{1}\right)$ is defined by the SPO of greenschist-facies minerals, with actinolitic amphibole $\left(\mathrm{Amp}_{1}\right)$ and chlorite as the main mineral phases. The 


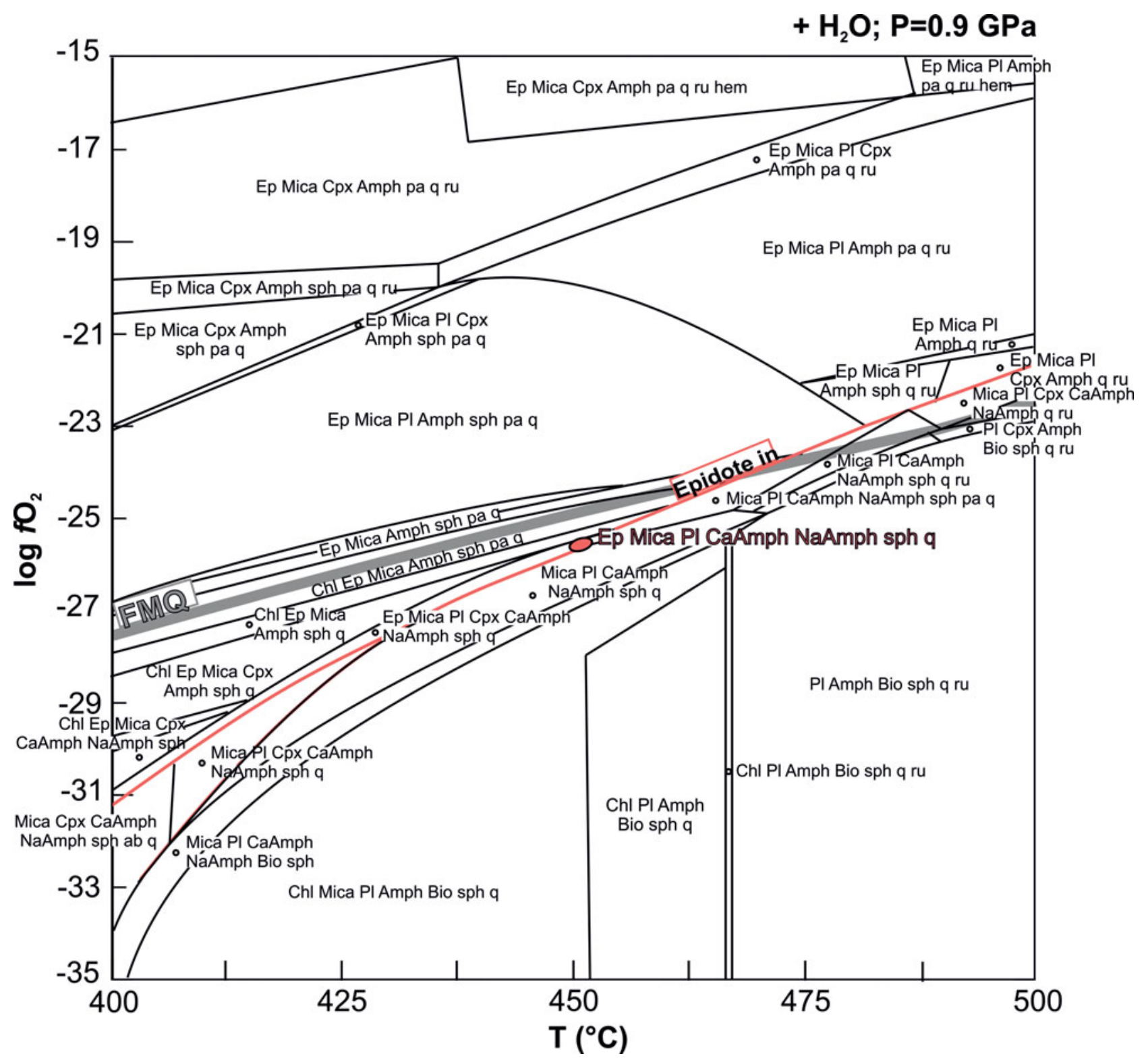

Figure 8. (Colour online) Isochemical $T-f \mathrm{O}_{2}$ section for Morghab meta-basalt calculated in the same system as that of Figure 7. The tiny red area shows the hypothetical stability field of the HP paragenesis. The red curve delineates the stability of epidote solid solution as a function of the oxidation state of the rock. The grey curve is the FMQ (fayalite-magnetite-quartz) reference redox buffer. Abbreviations are the same as Figure 7, except hem - haematite.

$\mathrm{S}_{1}$ fabric is overprinted by the static growth of sodic amphibole $\left(\mathrm{Amp}_{2}\right)$, white mica $\left(\mathrm{Wm}_{2}\right)$ with $\mathrm{Si}$ up to 3.56 apfu and a second generation of titanite $\left(\mathrm{Ttn}_{2}\right)$. The reconstructed recrystallization stages point to a $P-T$ evolution similar to the one shown by the CGC, with an early greenschist-facies stage followed by reequilibration at higher pressure (Fig. 9).

Isochemical $P-T$ pseudosections (Fig. 7) presented in this work and in Zanchi et al. (2015) suggest that the CGC and meta-basalts of the OC reached a similar pressure $(0.6-0.9 \mathrm{GPa})$ at temperatures slightly higher for the $\mathrm{OC}\left(390-440^{\circ} \mathrm{C}\right)$ with respect to the CGC $\left(320-380^{\circ} \mathrm{C}\right)$. Based on these considerations we can argue that the CGC mafic rocks and the OC meta-basalts experienced counter-clockwise $P-T$ paths with a first metamorphic stage that occurred at relatively HT and LP (greenschist facies), followed by a HP-LT (epidote blueschist facies) re-equilibration (Fig. 9).

The metamorphic gap between syn- $\mathrm{S}_{2}$ foliation phase assemblage of the MC (greenschist facies) and CGC (blueschist facies) indicates that the regional foliation in the two tectonometamorphic units formed at different ambient conditions.

The detailed microstructural and microprobe chemical analyses reveal different metamorphic stages characterizing the evolution of each AMC unit. A greenschist-facies metamorphism, likely occurring at low pressure as suggested by the absence of garnet and Na-clinopyroxene, has been individuated in the CGC and OC rocks. Such a stage is recorded by the occurrence of calcic amphiboles included in pre-main foliation porphyroblasts (CGC) or as the primary assemblage (OC) overprinted by the later static growth 

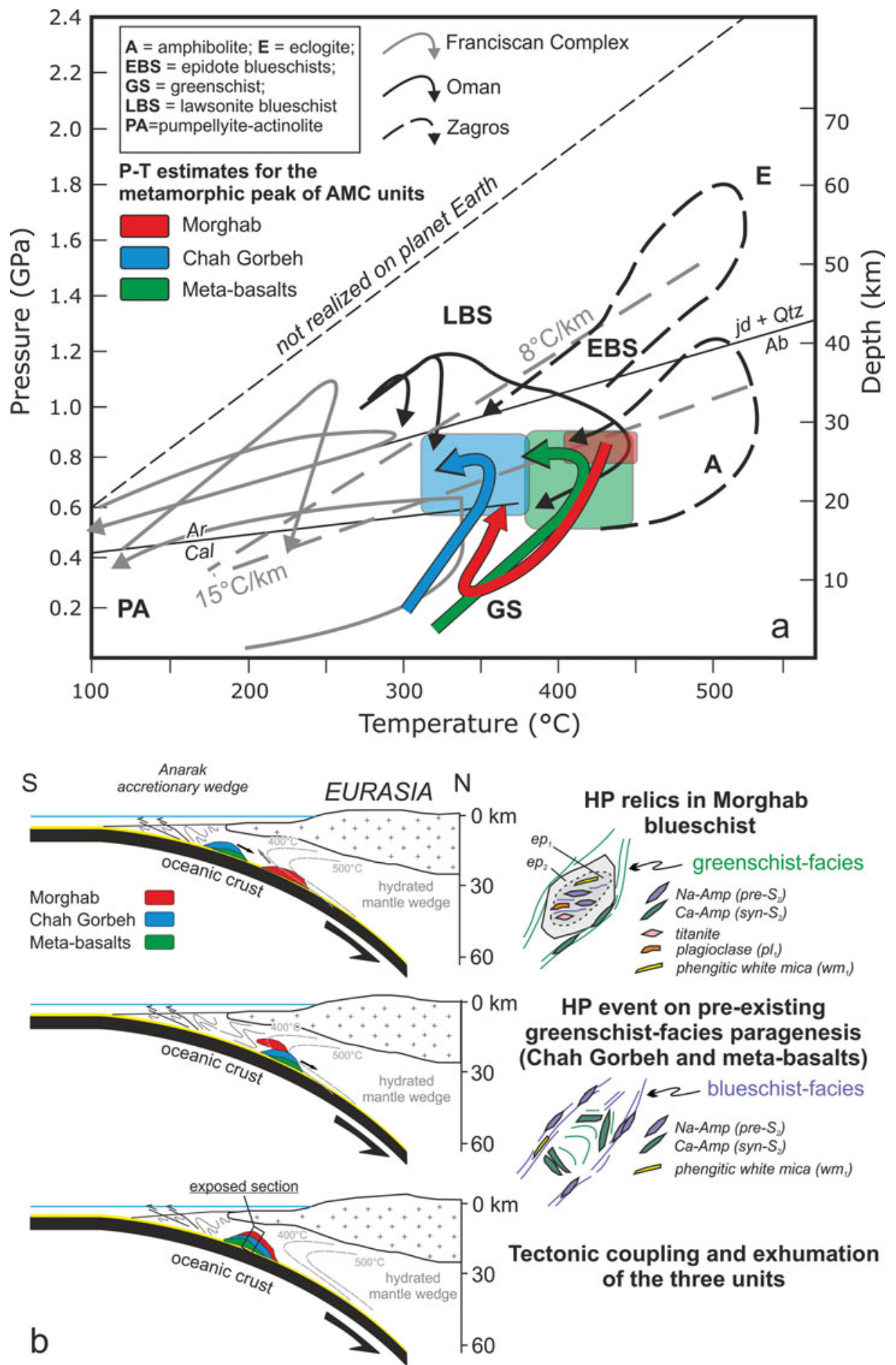

Figure 9. (Colour online) (a) $P-T$ estimates and inferred $P-T$ paths of the CGC, MC and OC blueschists. $P-T$ estimates for the GCG and OC metabasites are from Zanchi et al. (2015). Representative reconstructed $P-T$ paths for blueschists from the Franciscan subduction complex (Ernst, 1988; Banno et al. 2000; Ukar \& Cloos, 2014), Oman (Yamato et al. 2007; Agard et al. 2010) and Zagros (Angiboust et al. 2016) are reported for comparison. Metamorphic facies are from Evans (1990). (b) Cartoon depicting the suggest geodynamic scenario in which the tectonic units of the AMC acquired their contrasting $P-T$ evolutionary paths.

of HP-phase assemblages. This stage could be related to an early heating during subduction initiation, before the depression of the geotherms by continuous subduction of the oceanic crust. An alternative hypothesis is that this low-pressure stage is related to seafloor metamorphism overprinted during subduction. Ocean floor metamorphism is commonly associated with diffuse veining and intense metasomatism, which are both ab- sent in the AMC units. Quartz- and calcite-bearing veins occur in the pillow meta-basalts (Fig. 4d), but they cross-cut both pillows and sheared domains with a blueschist-facies overprint (Fig. 4b). This suggests that veining represents a late-stage feature, post-dating the burial and exhumation of the $\mathrm{OC}$ unit. The same holds true also for the mafic schist and associated rocks of the CGC (Zanchi et al. 2015). 
The counter-clockwise $P-T$ paths experienced by CGC and OC were likely due to early heating when they were underthrust beneath the southern Eurasia margin at the beginning of subduction (Fig. 9).

Such counter-clockwise $P-T$ paths are common within evolved accretionary wedges, when mafic rocks derived from the subducting oceanic crust or sediments scraped off the evolving prism are accreted in the early stages of subduction and later buried to increasing depth while geotherms are progressively depressed (Cloos, 1982).

On the other hand, the $P-T$ path of the $\mathrm{MC}$ is rather peculiar: an early HP-LT metamorphic stage is followed by greenschist-facies re-equilibration with a further pressure increase as suggested by amphibole and epidote compositional zoning. $P-T$ estimates made by forward thermodynamic modelling (fig. 24 in Zanchi et al. 2015) indicate similar pressures of the CGC and OC relative to the pre- $\mathrm{S}_{2}$ metamorphic peak, but at higher $T$ (Fig. 9). Dynamic metamorphism represented by the syn- $\mathrm{S}_{2}$ metamorphic stage re-equilibrated the HP-LT phase assemblages at greenschist-facies conditions. The chemical compositions (Fig. 6) of amphibole and epidote that grew during this greenschistfacies event point to a late- to post-deformation pressure increase. Summarizing, (Table 2) the MC mafic rocks experienced a clockwise $P-T$ path, with an early HP-LT stage followed by exhumation at lower depths (greenschist-facies re-equilibration) and subsequent pressure increase likely due to re-subduction within the accretionary wedge.

Despite the possible difference in $P-T$ paths experienced by the three units, a temperature/depth gradient of $c .15^{\circ} \mathrm{C} \mathrm{km}^{-1}$ seems to be the best estimate for the peak pressure in all units (Fig. 9).

Counter-clockwise $P-T$ paths within exhumed accretionary prisms are well known for block-in-matrix subduction mélanges, like the Franciscan Complex (Ukar, 2012; Ukar \& Cloos, 2014), but are less common in HP-LT complexes made of coherent tectonic units. The Hajiabad blueschists of the Zagros orogen (Agard et al. 2006; Angiboust et al. 2016) display instead such features (Fig. 9). Despite different metamorphic peak conditions, several units underwent an initial heating stage during underplating, followed by HP-LT dynamic metamorphism. Geochronological data suggest that tectonic units reached pressure peak at different times (Angiboust et al. 2016). The occurrence and preservation of counter-clockwise $P-T$ paths are likely favoured for tectonic units that were underplated in the first subduction stages, along a relatively hot $\left(15-17^{\circ} \mathrm{C} \mathrm{km}^{-1}\right)$ geothermal gradient. Geotherms are then progressively depressed as the subduction zone evolves and early subducted units can be exhumed along a cold $\left(<10^{\circ} \mathrm{C} \mathrm{km}^{-1}\right)$ subduction channel.

The age of HP-LT metamorphism in the AMC units is debated. Bagheri \& Stampfli (2008) proposed an Early Permian age (285 Ma) for blueschists of the CGC, and a 'Variscan' age for the HP-LT metamorph- ism of the MC, suggesting that the two units formed in distinct times and palaeotectonic settings. However, these geochronological data are highly debatable (Zanchi et al. 2015) as microstructural and chemical features of the dated minerals are loosely constrained. To our knowledge metamorphism and deformation of AMC units should be constrained to pre-Sakmarian (c. $300 \mathrm{Ma}$ or older) times (Zanchi et al. 2015) and no robust data exist on the age of HP-LT metamorphism. Even if we take as valid the available geochronological data of the HP-LT metamorphism of the AMC, we need to consider that in the context of a long-lasting subduction (e.g. several tens of millions of years) the subducted rocks could reach the metamorphic peak at different ambient conditions and at different times (e.g. Gerya, Stockhert \& Perchuk, 2002; Ukar, 2012). Therefore, the meta-basalts and the blueschists of the CGC and MC, even if they recorded contrasting $P-T$ evolutions and were possibly metamorphosed at different ages, could still be considered as parts of a single accretionary complex.

The nature and provenance of the continental blocks that collided with the southern Eurasian margin is also controversial. The Anarak block strongly differs from the Upper Palaeozoic successions of Central and North Iran that record an extensional evolution related to the opening of the Neo-Tethys that caused the northward drift of Iran (Angiolini et al. 2007; Gaetani et al. 2009). A possible provenance, discussed in Zanchi et al. (2015), is that the AMC was part of the accretionary wedge developed above the Palaeo-Tethys subduction zone. In NE Iran, the Variscan-Cimmerian complexes of Binalood and Fariman (Sheikholeslami \& Kouhpeyma, 2012; Zanchetta et al. 2013) recorded the upper plate history of the Palaeo-Tethys subduction. The AMC could be originally placed to the SW of the Fariman complex and later shifted westwards to its present-day position in the framework of the large counter-clockwise rotation along a vertical axis that affected Central Iran from Triassic time (Muttoni et al. 2009; Mattei et al. 2015).

\section{Conclusions}

The AMC of Central Iran is made of several units among which three of them, the Morghab, Chah Gorbeh and meta-basalts complexes, have been metamorphosed at HP-LT conditions within the blueschist facies.

At least three deformation events have been recognized in the MC and CGC. The second deformation stage is responsible for the development of the regional foliation $\left(\mathrm{S}_{2}\right)$ that is generally concordant in the two units. Microstructural investigations and microprobe analyses demonstrated that the main foliation developed at blueschist-facies and greenschist-facies conditions for the CGC and MC units, respectively. The main fabric element of the CGC and MC thus formed when the two units were at different depths within the accretionary wedge. 
The reconstruction of the deformationmetamorphism relationships and $P-T$ estimates of the metamorphic stages allowed the definition of a clockwise $P-T$ path for the $\mathrm{MC}$ and a counterclockwise path for CGC and meta-basalts of the OC. $P-T$ conditions at metamorphic peak were of 410 $450{ }^{\circ} \mathrm{C}$ at $0.78-0.9 \mathrm{GPa}$ for the $\mathrm{MC}, 390-440^{\circ} \mathrm{C}$ at $0.6-0.9 \mathrm{GPa}$ for the meta-basalts and $320-380^{\circ} \mathrm{C}$ at 0.6-0.9 GPa for the CGC.

The presented data suggest that the three units reached almost the same depth $(c .25 \mathrm{~km})$ within the Anarak accretionary wedge, but followed different subduction-exhumation paths that resulted in a contrasting $P-T$ evolution.

Acknowledgements. This paper results from the PRIN 2010/2011 Project BIRTH AND DEATH OF OCEANIC BASINS: GEODYNAMIC PROCESSES FROM RIFTING TO CONTINENTAL COLLISION IN MEDITERRANEAN AND CIRCUM-MEDITERRANEAN OROGENS and the DARIUS PROGRAMME. We are grateful to Vachik Hairapetian (Department of Geology, Esfahan Branch, Islamic Azad University, Iran) for providing us information on the age of the Lakh Marble. Comments and suggestions by Yann Rolland and an anonymous reviewer helped us to improve the quality of the paper. G. Capponi is warmly thanked for the editorial handling of the paper. We are grateful to the Geological Survey of Iran (Teheran branch) for its continuous support during several years of common research activities in Central Iran.

\section{References}

Agard, P., Monié, P., Gerber, W., Omrani, J., Molinaro, M., Meyer, B. \& Yamato, P. 2006. Transient, synobduction exhumation of Zagros blueschists inferred from $P-T$, deformation, time, and kinematic constraints: implications for Neotethyan wedge dynamics. Journal of Geophysical Research: Solid Earth 111 B11401, doi: 10.1029/2005JB004103.

Agard, P., Searle, M. P., Alsop, G. I. \& DubacQ, B. 2010. Crustal stacking and expulsion tectonics during continental subduction: $\mathrm{P}-\mathrm{T}$ deformation constraints from Oman. Tectonics 29, TC5018, doi: 10.1029/2010TC002669.

Angiboust, S., Agard, P., Glodny, J., Omrani, J. \& ONCKEN, O. 2016. Zagros blueschists - episodic underplating and long-lived cooling of a subduction zone. Earth and Planetary Science Letters 443, 48-58.

Angiolini, L., Gaetani, M., Muttoni, G., Stephenson, M. H. \& ZANCHI, A. 2007. Tethyan oceanic currents and climate gradients 300 my ago. Geology 35, 1071-4.

BAGHERI, S. \& StAmpfli, G. M. 2008. The Anarak, Jandaq and Posht-e-Badam metamorphic complexes in central Iran: new geological data, relationships and tectonic implications. Tectonophysics 451, 123-55.

Balini, M., Nicora, A., Berra, F., Garzanti, F., Levera, M., Mattei, M., Muttoni, M., Zanchi, A., Bollati, I., Larghi, C., Zanchetta, S., SAlamati, R. \& Mossavvari, F. 2009. The Triassic stratigraphic succession of Nakhlak (central Iran), a record from an active margin. In South Caspian to Central Iran Basins (eds M. F. Brunet, M. Wilmsen \& J. W. Granath), pp. 287321. Geological Society of London, Special Publication no. 312 .
Banno, S., Shibakusa, H., Enami, M., Wang, C.-L. \& ERnst, W. G. 2000. Chemical fine structure of Franciscan jadeitic pyroxene from Ward Creek, Cazadero area, California. The American Mineralogist 85, 1795-8.

Berra, F., Zanchi, A., Angiolini, L., Vachard, D., Vezzoli, G., Zanchetta, S., Bergomi, M., Javadi, H. R., Kounpeyma, M. 2017. The upper Palaeozoic Godar-e-Siah Complex of Jandaq: evidence and significance of a North Palaeotethyan succession in Central Iran. Journal of Asian Earth Sciences 138, 272-90.

Buchs, D. M., Bagheri, S., Martin, K., Hermann, J. \& Arculus, R. 2013. Paleozoic to Triassic ocean opening and closure preserved in Central Iran: constraints from the geochemistry of meta-igneous rocks of the Anarak area. Lithos 172-173, 267-87.

Cloos, M. 1982. Flow mélange: numerical modelling and geologic constrain on their origin in the Franciscan subduction complex, California. Geological Society of America Bulletin 93, 330-44.

Cloos, M. \& Shreve, R. L. 1988a. Subduction-channel model of prism accretion, mélange formation, sediment subduction, and subduction erosion at convergent plate margins: 1. background and description. Pure and Applied Geophysics 128, 455-500.

Cloos, M. \& Shreve, R. L. 1988b. Subduction-channel model of prism accretion, mélange formation, sediment subduction, and subduction erosion at convergent plate margins: 2. implications and discussion. Pure and Applied Geophysics 128, 501-45.

ConNolly, J. A. D. 2005. Multivariable phase diagrams: an algorithm based on generalized thermodynamics. American Journal of Science 290, 666-718.

Dale, J., Powell, R., White, R. W., Elmer, F. L. \& Holland, T. J. B. 2005. A thermodynamic model for $\mathrm{Ca}-\mathrm{Na}$ clinoamphiboles in $\mathrm{Na} 2 \mathrm{O}-\mathrm{CaO}-\mathrm{FeO}-\mathrm{MgO}-$ $\mathrm{Al} 2 \mathrm{O} 3-\mathrm{SiO} 2-\mathrm{H} 2 \mathrm{O}-\mathrm{O}$ for petrological calculations. Journal of Metamorphic Geology 23, 771-91.

ERnst, W. G. 1973. Blueschist metamorphism and $P-T$ regimes in active subduction zones. Tectonophysics $\mathbf{1 7}$, 255-72.

ERnst, W. G. 1988. Tectonic history of subduction zones inferred from retrograde blueschist $P-T$ paths. Geology 16, 1081-4.

Evans, B. W. 1990. Phase relations of epidote-blueschists. Lithos 35, 3-23.

Gaetani, M., Angiolini, L., Ueno, K., Nicora, A., Stephenson, M. H., Sciunnach, D., Rettori, R., Price, G. D. \& SAbouri, J. 2009. Pennsylvanian-Early Triassic stratigraphy in the Alborz Mountains (Iran). In South Caspian to Central Iran Basins (eds M. F. Brunet, M. Wilmsen \& J. W. Granath), pp. 79-128. Geological Society of London, Special Publication no. 312.

GerYa, T. \& Stockhert, B. 2006. Two-dimensional numerical modeling of tectonic and metamorphic histories at active continental margins. International Journal of Earth Sciences 95, 250-74.

Gerya, T. V., Stockhert, B. \& Perchuk, A. L. 2002. Exhumation of high-pressure metamorphic rocks in a subduction channel: a numerical simulation. Tectonics $\mathbf{2 1}$, 6-1-6-19.

Ghasemi, A. M. \& Talbot, C. J. 2006. A new tectonic scenario for the Sanandaj-Sirjan Zone (Iran). Journal of Asian Earth Sciences 26, 683-93.

Grapes, R. H. \& Hoskin, P. W. 2004. Epidote group minerals in low-medium pressure metamorphic terranes. In Epidotes (eds A. Liebscher \& G. Franz), pp. 301-55. Reviews in Mineralogy and Geochemistry 56. 
Holland, T. J. B. \& Powell, R. 1998. An internally consistent thermodynamic data set for phases of petrologic interest. Journal of Metamorphic Geology 16, 309-43.

Holland, T. J. B. \& Powell, R. 2003. Activity-composition relations for phases in petrological calculations: an asymmetric multicomponent formulation. Contributions to Mineralogy and Petrology 145, 492-501.

Hsu, K. J. 1968. Principles of mélanges and their bearing on the Franciscan-Knoxville paradox. Geological Society of America Bulletin 79, 1063-74.

Kress, V. C. \& CARmichael, I. S. E. 1988. Stoichiometry of the iron oxidation reaction in silicate melts. American Mineralogist 73, 1267-74.

Kruse, P. D. \& Zhuralev, A. Yu. 2008. Middle-Late Cambrian Rankenella-Girvanella reefs of the Mila Formation Northern Iran. Canadian Journal of Earth Sciences 45, 619-39.

Mattei, M., Cifelli, F., Muttoni, G. \& Rashid, H. 2015. Post-Cimmerian (Jurassic-Cenozoic) paleogeography and vertical axis rotation of Central Iran and the Alborz Mountains. Journal of Asian Earth Sciences 102, 92-101.

Mel'nikov, B. N., Rozanov, A. Yu., Susov, M. V. \& Fonin V., D. 1986. Pervye arkheotsiaty iz nizhengo kembriya tsentral'nogo Irana (First archaeocyaths from the Lower Cambrian of central Iran). Izvestiya, Akademiya Nauk SSSR 7, 134-8.

Muttoni, M., Mattei, M., Balini, M., Zanchi, A., GaETANi, M. \& BerRa, F. 2009. The drift history of Iran from the Ordovician to the Triassic. In South Caspian to Central Iran Basins (eds M. F. Brunet, M. Wilmsen \& J. W. Granath), pp. 7-29. Geological Society of London, Special Publication no. 312.

ŞENGör, A. M. C. 1979. Mid-Mesozoic closure of Tethys and its implications. Nature 279, 590-3.

Sharkovski, M., Susov, M. \& Krivyakin, B. 1984. Geology of the Anarak Area (Central Iran). Explanatory Text of the Anarak Quadrangle Map 1:250000. Geological Survey of Iran, V/O "Tecnoexport" USSR Ministry of Geology Reports, 19.

Sheikholeslami, M. R. \& Kouhpeyma, M. 2012. Structural analysis and tectonic evolution of the eastern
Binalud Mountains, NE Iran. Journal of Geodynamics 61, 23-46.

ToRABI, G. 2012. Late Permian post-ophiolitic trondhjemites from Central Iran: a mark of subduction role in growth of Paleozoic continental crust. Island Arcs 21, 215-29.

UKAR, E. 2012. Tectonic significance of low-temperature blueschists blocks in the Franciscan mélange at san Simeon, California. Tectonophysics 568-569, 154-69.

UKar, E. \& Cloos, M. 2014. Low-temperature blueschistfacies mafic blocks in the Franciscan mélange, San Simeon, California. Geological Society America Bulletin, B30876.1, doi: 10.1130/B30876.1.

WiLson, A. D. 1955. Determination of ferrous iron in rocks and minerals. Bulletin of the Geological Survey of Great Britain 9, 56-8.

Yamato, P., Agard, P., Goffé, B., De Andrade, V., Vidal, O. \& Jolivet, L. 2007. New, high precision P-T estimates for Oman blueschists: implications for obduction, nappe stacking and exhumation processes. Journal of Metamorphic Geology 25, 657-82.

Yokoyama, T. \& Nakamura, E. 2002. Precise determination of ferrous iron in silicate rocks. Geochemica and Cosmochemica Acta 66, 1085-93.

Zanchetta, S., Berra, F., Zanchi, A., Bergomi, M., Caridroit, M., Nicora, M. \& Heidarzadeh, G. 2013. The record of the Late Palaeozoic active margin of the Palaeotethys in NE Iran: constraints on the Cimmerian orogeny. Gondwana Research 24, 1237-66.

Zanchi, A., Malaspina, N., Zanchetta, S., Berra, F., Benciolini, L., Bergomi, M., Cavallo, A., Javadi, H. R. \& Kounpeyma, M. 2015. The Cimmerian accretionary wedge of Anarak, Central Iran. Journal of Asian Earth Sciences 102, 45-72.

Zanchi, A., Zanchetta, S., Garzanti, E., Balini, M., Berra, F., Mattei, M. \& Muttoni, G. 2009. The Cimmerian evolution of the Nakhlak-Anarak area, central Iran, and its bearing for the reconstruction of the history of the Eurasian margin. In South Caspian to Central Iran Basins (M. F. Brunet, M. Wilmsen \& J. W. Granath, eds), pp. 261-86. Geological Society of London, Special Publications no. 312 . 